\title{
Long non-coding RNA MAPKAPK5-AS1/ PLAGL2/HIF-1a signaling loop promotes hepatocellular carcinoma progression
}

Liang Wang, Liankang Sun, Runkun Liu, Huanye Mo, Yongshen Niu, Tianxiang Chen, Yufeng Wang, Shaoshan Han, Kangsheng Tu* (D) and Qingguang Liu*

\begin{abstract}
Background: Long non-coding RNAs (IncRNAs) are widely involved in human cancers' progression by regulating tumor cells' various malignant behaviors. MAPKAPK5-AS1 has been recognized as an oncogene in colorectal cancer. However, the biological role of MAPKAPK5-AS1 in hepatocellular carcinoma (HCC) has not been explored.

Methods: Quantitative real-time PCR was performed to detect the level of MAPKAPK5-AS1 in HCC tissues and cell lines. The effects of MAPKAPK5-AS1 on tumor growth and metastasis were assessed via in vitro experiments, including MTT, colony formation, EdU, flow cytometry, transwell assays, and nude mice models. The western blotting analysis was carried out to determine epithelial-mesenchymal transition (EMT) markers and AKT signaling. The interaction between MAPKAPK5-AS1, miR-154-5p, and PLAGL2 were explored by luciferase reporter assay and RNA immunoprecipitation. The regulatory effect of HIF-1a on MAPKAPK5-AS1 was evaluated by chromatin immunoprecipitation.

Results: MAPKAPK5-AS1 expression was significantly elevated in HCC, and its overexpression associated with malignant clinical features and reduced survival. Functionally, MAPKAPK5-AS1 knockdown repressed the proliferation, mobility, and EMT of HCC cells and induced apoptosis. Ectopic expression of MAPKAPK5-AS1 contributed to HCC cell proliferation and invasion in vitro. Furthermore, MAPKAPK5-AS1 silencing suppressed, while MAPKAPK5-AS1 overexpression enhanced HCC growth and lung metastasis in vivo. Mechanistically, MAPKAPK5-AS1 upregulated PLAG1 like zinc finger 2 (PLAGL2) expression by acting as an endogenous competing RNA (ceRNA) to sponge miR-154-5p, thereby activating EGFR/AKT signaling. Importantly, rescue experiments demonstrated that the miR-154-5p/PLAGL2 axis mediated the function of MAPKAPK5-AS1 in HCC cells. Interestingly, we found that hypoxia-inducible factor 1a (HIF-1a), a transcript factor, could directly bind to the promoter to activate MAPKAPK5AS1 transcription. MAPKAPK5-AS1 regulated HIF-1a expression through PLAGL2 to form a hypoxia-mediated MAPK APK5-AS1/PLAGL2/HIF-1a signaling loop in HCC.
\end{abstract}

Conclusions: Our results reveal a MAPKAPK5-AS1/PLAGL2/HIF-1 a signaling loop in HCC progression and suggest that MAPKAPK5-AS1 could be a potential novel therapeutic target of HCC.

Keywords: MAPKAPK5-AS1, miR-154-5p, PLAGL2, Hepatocellular carcinoma progression, Hypoxia

\footnotetext{
* Correspondence: tks0912@foxmail.com; liuqingguang@vip.sina.com Department of Hepatobiliary Surgery, The First Affiliated Hospital of Xi'an Jiaotong University, 277 Yanta West Road, Xi'an 710061, China
} 


\section{Background}

Hepatocellular carcinoma (HCC), as the most common type of liver cancer, is the third leading cause of tumorrelated deaths worldwide [1]. Despite advances made in HCC treatment, surgical resection is still the main treatment method. However, the prognosis of HCC patients is still not satisfactory, and the reason is mainly due to metastasis and recurrence after surgical removal of the tumor [2]. The exact mechanism of tumor occurrence and metastasis remain largely unclear. Therefore, it is very urgent and essential to clarify tumorigenesis and metastasis's molecular mechanism and develop new treatments for HCC.

In recent years, numerous long non-coding RNAs (lncRNAs) have been discovered and proved by many studies to be involved in the progression of various tumors, including HCC [3-6]. For example, Zhe Li et al. reported that LINC00624 enhances liver cancer progression by disrupting the histone deacetylase 6 (HDAC6)tripartite motif containing 28 (TRIM28) - zinc finger potein 354C (ZNF354C) corepressor complex [6]. Hui He et al. confirmed that lncRNA ZFPM2-AS1 acts as a miRNA sponge and promotes cell invasion through regulation of miR-139/growth differentiation factor 10 (GDF10) in HCC [7]. Our research team also discovered and studied the role of several lncRNAs in the progression of HCC in previous studies. For instance, we found that lncRNA CASC2 suppresses epithelial-mesenchymal transition of hepatocellular carcinoma cells through the CASC2/miR-367/ F-box and WD repeat domain containing 7 (FBXW7) axis [8]. Moreover, our study confirmed that MCM3AP-AS1 promotes hepatocellular carcinoma growth by targeting the miR-194-5p/ forkhead box A1 (FOXA1) axis [9]. Additional, the biological roles of lncRNA DSCR8 [10], AGAP2-AS1 [11], RUNX1-IT1 [12], and PICSAR [13] in HCC were investigated by our team. Here, we identified a novel upregulated lncRNA, MAPK APK5-AS1, in HCC using The Cancer Genome Atlas (TCGA) data and Gene Expression Omnibus (GEO) datasets, and TCGA data also showed that MAPKAPK5-AS1 predicted poor prognosis of HCC patients. However, the expression, function, and exact mechanism of MAPK APK5-AS1 is unknown.

Extensive work has reported that hypoxia, a hallmark of the solid tumor microenvironment, is involved in the progression of various cancers, including HCC [14-16]. Hypoxia-inducible factor $1 \alpha$ (HIF-1 $\alpha$ ), the key molecule in response to hypoxia, as a transcription factor, regulates the expression of a series of hypoxia-responsive genes, thereby mediating the effect of hypoxia on the biological behavior of cells [14, 17-20]. Recently, the effect of hypoxia on IncRNAs has attracted widespread attention, and increasing hypoxia-responsive lncRNAs are identified and studied. Libin Qiu et al. found that
IncRNA DANCR alleviates hypoxia-caused H9c2 cells damage through the up-regulation of HIF-1 $\alpha$ [21]. Lina Dong et al. confirmed that a positive feedback loop of lncRNA DSCR8/miR-98-5p/STAT3/HIF-1 $\alpha$ plays a role in ovarian cancer [22]. Mingxing $\mathrm{Hu}$ et al. demonstrated that lncRNA HOTAIR knockdown inhibits glycolysis by regulating miR-130a-3p/ HIF- $1 \alpha$ in HCC under hypoxia [23]. We also found that IncRNA RUNX1-IT1 downregulated by hypoxia-driven histone deacetylase 3 represses proliferation and cancer stem-like properties in HCC cells [12]. However, the effect of hypoxia on MAPK APK5-AS1 expression is not explored.

In the present study, the expression level of MAPK APK5-AS1 was detected. Subsequently, a series of in vitro and in vivo functional assays were carried out to investigate the biological roles of MAPKAPK5-AS1 in HCC. Next, the downstream mechanisms that mediate the functions of MAPKAPK5 were screened and verified. Finally, the effect of hypoxia on MAPKAPK5-AS1 expression was studied.

\section{Methods \\ Tissue samples}

Tissue samples used in this study were collected from 97 HCC patients at the First Affiliated Hospital of Xi'an Jiaotong University (Xi'an, China) between September 2014 to August 2015. Tumor and adjacent non-tumor tissue samples were immediately frozen in liquid nitrogen after being obtained and subsequently maintained in $-80^{\circ} \mathrm{C}$. The clinicopathological features of HCC patients were exhibited in Table 1.

\section{Cell lines and cell culture}

The human immortalized normal hepatic cell line (L02), HCC cell lines (Huh7, Hep3B, MHCC97-H, MHCC97-L, HepG2, HCCLM3), and HEK293T cells were obtained from the Cell Bank, Type Culture Collection, Chinese Academy of Sciences (Shanghai, China). All cells were cultured in Dulbecco's modified Eagle medium (DMEM) (Gibco, Grand Island, NY, USA) containing 10\% fetal bovine serum (FBS) (Gibco, Grand Island, NY, USA) and $1 \%$ penicillin-streptomycin (Sigma, St-Louis, MO, USA). All cell lines were cultured in a humidified incubator containing $5 \% \mathrm{CO}_{2}$ at $37^{\circ} \mathrm{C}$. Hypoxia incubator $(1 \% \mathrm{O} 2)$ was used to establish the hypoxic cell model.

\section{Cell transfection}

sh-MAPKAPK5-AS1 (shRNA\#1,2,3) and sh-negative control (sh-control) lentiviruses were purchased from GeneCreate Biological Engineering Co., Ltd. (Wuhan, China). The expression vector pcDNA3.1(+) (MAPKAPK5AS1) and the empty plasmid pcDNA3.1(+) (Vector) were obtained from Invitrogen Corporation (Carlsbad, CA, USA). Hsa-miR-154-5p mimic (miR10000452-1-5) and 
Table 1 Association between MAPKAPK5-AS1 expression and clinicopathologic features of hepatocellular carcinoma patients

\begin{tabular}{|c|c|c|c|c|}
\hline \multirow[t]{2}{*}{ Characteristics } & \multirow[t]{2}{*}{$n=97$} & \multicolumn{2}{|c|}{ MAPKAPK5-AS1 levels } & \multirow[t]{2}{*}{$p$-value } \\
\hline & & High $(n=49)$ & Low $(n=48)$ & \\
\hline Age (years) & & & & 0.774 \\
\hline$\geq 60$ & 64 & 33 & 31 & \\
\hline$<60$ & 33 & 16 & 17 & \\
\hline Gender & & & & 0.255 \\
\hline Male & 81 & 43 & 38 & \\
\hline Female & 16 & 6 & 10 & \\
\hline HBV infection & & & & 0.184 \\
\hline Negative & 19 & 7 & 12 & \\
\hline Positive & 78 & 42 & 36 & \\
\hline Liver cirrhosis & & & & 0.240 \\
\hline Absent & 29 & 12 & 17 & \\
\hline Present & 68 & 37 & 31 & \\
\hline AFP (ng/ml) & & & & 0.305 \\
\hline$\leq 20$ & 22 & 9 & 13 & \\
\hline$>20$ & 75 & 40 & 35 & \\
\hline Tumor size & & & & $0.006^{*}$ \\
\hline$\leq 5 \mathrm{~cm}$ & 41 & 14 & 27 & \\
\hline$>5 \mathrm{~cm}$ & 56 & 35 & 21 & \\
\hline Tumor multiplicity & & & & $0.001^{*}$ \\
\hline Single & 72 & 29 & 43 & \\
\hline Multiple & 25 & 20 & 5 & \\
\hline Vascular invasion & & & & $0.008^{*}$ \\
\hline No & 60 & 24 & 36 & \\
\hline Yes & 37 & 25 & 12 & \\
\hline TNM stage & & & & $0.001^{*}$ \\
\hline $1+\|$ & 78 & 33 & 45 & \\
\hline$I I I+I V$ & 19 & 16 & 3 & \\
\hline
\end{tabular}

* indicates statistically significant

hsa-miR-154-5p inhibitor (miR20000452-1-5) were purchased from RiboBio (Guangzhou, China). PLAGL2 Human cDNA ORF Clone (PLAGL2) and PLAGL2 siRNA (si-PLAGL2) were purchased from Technologies, Inc. (USA). The HIF-1 $\alpha$ siRNA was purchased from Shanghai GenePharma Co., Ltd. (Shanghai, China). Cell transfection was performed according to the manufactures' instructions.

\section{Quantitative real-time polymerase chain reaction (qRT-}

PCR)

TRIzol (Invitrogen) was used to extract total RNA from HCC tissues and cells following the manufacturer's procedure. All miRNAs were reverse transcribed into cDNA with the miDETECT A Track miRNA qRT-PCR Starter Kit (C10712-1, RiboBio, Guangzhou, China). All lncRNAs and mRNAs were reverse transcribed into
cDNA with the RevertAid First Strand cDNA Synthesis Kit (Thermo-Fisher Scientific). All primers used in this study were customized from RiboBio, and the sequence of primers are listed in Additional File 1: Table S1.

\section{MTT assay}

Cells were added in a 96-well plate at a density of $1 \times$ $10^{4} / \mathrm{ml}$. $20 \mu \mathrm{l}$ MTT solution (Sigma, St. Louis, MO, USA) was added to each well after incubation for 24,48 , 72 , and $96 \mathrm{~h}$. Then, the cells continued to be cultured for $4 \mathrm{~h}$ in a humidified incubator. After the supernatants were removed, $200 \mu \mathrm{l}$ DMSO was added to each well. Finally, the absorbance was detected with a microplate reader (Bio-Rad, Hercules, CA, USA).

\section{Colony formation assay}

Cells transfected for $48 \mathrm{~h}$ were plated in 6-well plates $\left(1 \times 10^{3} /\right.$ well). After cultured for 14 days, cell colonies were first fixed with $4 \%$ paraformaldehyde for $20 \mathrm{~min}$, then stained with $0.5 \%$ crystal violet for $20 \mathrm{~min}$, and finally photographed.

\section{Ethynyl deoxyuridine incorporation assay}

Cell-Light EdU Apollo567 kit (Guangzhou RiboBio Co., Ltd.) was used to assess cell proliferation, and Zeiss fluorescence photomicroscope (Carl Zeiss, Oberkochen, Germany) was applied to acquire the results. EdU positive rate is calculated by counting at least five random fields.

\section{Cell apoptosis analysis}

FITC Annexin V Apoptosis Detection Kit I was used to measure cell apoptosis in the study. And the assay was performed according to the manufactures' instructions.

\section{Transwell assay}

Transwell migration and invasion assays were conducted to evaluated cell mobility. These assays were conducted according to the protocols described in our previous studies [24].

\section{Western blotting}

Protein extraction and western blotting assay were conducted according to the protocols described in our previous studies [24]. The primary antibodies used in this study are listed in Additional File 2: Table S2.

\section{Animal models}

Subcutaneous xenograft model and lung metastasis model were established to assess the growth and metastasis ability of cells in vivo. Male BALB/C nude mice (4-weekold) were purchased from Shanghai SLAC Laboratory Animal Center of Chinese Academy of Sciences (Shanghai, China) and randomly divided into four groups $(n=6$ per 
group). HCCLM3 cells transfected with sh-MAPKAPK5AS1 and sh-control and Hep3B transfected with MAPK APK5-AS1 and Vector were applied in our animal experiments. For subcutaneous xenograft model, transfected HCCLM3 and Hep3B cells were subcutaneously injected into the left flank of the mice, respectively $\left(1 \times 10^{6}\right.$ per injection) (six mice per group). Tumor size was measured every 7 days, and tumor volume was calculated by the formula: tumor volume $=0.5 \times($ long diameter $) \times($ short diameter) $)^{2}$. Tumors were removed for measurement after 4 weeks. For lung metastasis model, transfected HCCLM3 and $\mathrm{Hep} 3 \mathrm{~B}$ cells were injected via the tail vein $\left(1 \times 10^{6}\right.$ per injection) (five mice per group). After 6 weeks, mice were sacrificed and the lung metastases were confirmed by hematoxylin and eosin (H\&E) staining. The animal experiments were approved by the Animal Care and Use Committee of Xi'an Jiaotong University.

\section{Immunohistochemistry (IHC)}

After the paraffin-embedded tumor tissue was sectioned, IHC staining for Ki67, E-Cadherin, N-Cadherin, and Vimentin was performed to assess the levels of the proteins according to the experimental instructions. Finally, the slides were scanned with the Leica slide scanning microscope imaging system. The result of Ki67 was assessed by the percentage of positive staining cells. The staining of epithelial-mesenchymal transition (EMT) markers were evaluated by IHC scores. The scoring is based on staining intensity: no staining scored 0 , weakly staining scored 1 , moderately staining scored 2 , and strongly staining scored 3. The final score was calculated using the following percentage score $\times$ staining intensity score.

\section{Subcellular fractionation}

The nuclear and cytoplasmic separation was performed with the PARIS Kit (Life Technologies, USA) according to the manufacturer's instructions.

\section{Luciferase reporter assays}

The wild-type (wt) or mutant (mut) sequence of MAPK APK5-AS1 or 3'UTR of PLAGL2 was cloned into pGL3 vector (Promega, Madison, WI, USA), separately. The wt or mut luciferase plasmids and miRNA were co-transfected into HEK293T cells. The MAPKAPK5-AS1 promoters containing different HREs are cloned into pGL3-basic vector, and the pGL3-MAPKAPK5-AS1vectors were cotransfected with si-control or si-HIF-1 $\alpha$ under hypoxia. According to the manufacturer's instructions, the luciferase activity was detected with a Dual-Luciferase Reporter Assay System (Promega, USA) $48 \mathrm{~h}$ after transfection.

\section{Ago2-RNA immunoprecipitation (RIP) assay}

The EZ-Magna RIP Kit (Millipore, Bedford, MA, USA) was used to performed RIP assay to explore the intracellular state of MAPKAPK5-AS1 and miR-154-5p. Anti-Ago2 and control IgG (Millipore, USA) antibodies were used in the assay. The coprecipitated MAPKAPK5-AS1 or miR-154-5p was evaluated by qRT-PCR.

\section{Chromatin Immunoprecipitation (ChIP)}

ChIP assays were performed using a ChIP kit (CST, USA) to investigate the intracellular combination between HIF1 and HREs of MAPKAPK5-AS1 promoter. Cells were crosslinked with formaldehyde and sonicated to an average length of 200-1000 bp. Then, Immunoprecipitation was conducted with an anti-HIF- $1 \alpha$ antibody (Abcam) or IgG control. Precipitated DNA was amplified by qRT-PCR using primers listed in Additional File 1: Table S1.

\section{Statistical analysis}

Data of the study were shown as means \pm SD, and at least three independent replicates were conducted. The differences were analyzed by Student's $t$-test and analysis of variance using GraphPad Prism 7.0 (San Diego, CA, USA) and SPSS software 24.0 (SPSS Inc., Chicago, IL, USA). Statistical significance was evaluated via the Kaplan-Meier method, the log-rank test, and Pearson's correlation analysis. $p<0.05$ was deemed to indicate statistical significance.

\section{Results \\ The differential expression and clinical significance of MAPKAPK5-AS1 in HCC}

GEO and TCGA public databases were analyzed to identify lncRNAs which potentially exert vital biological function in HCC progression, and lncRNA MAPKAPK5AS1 which was consistently elevated in HCC in three public databases, TCGA database $(p<0.0001$, Fig. 1a), GSE45436 $(p<0.0001$, Fig. 1b), and GSE54236 $(p<$ 0.0001, Additional File 3: Fig. S1A), interested us. Subsequently, our qRT-PCR results confirmed the high expression of MAPKAPK5-AS1 in HCC tissues compared to adjacent non-tumor tissues $(p<0.0001$, Fig. 1c). Furthermore, compared with human normal liver cells (L02), the MAPKAPK5-AS1 expression level was significantly upregulated in HCC cell lines (all $p<0.05$, Additional File 3: Fig. S1B). Next, based on median MAPK APK5-AS1expression in HCC tissues, patients were divided into MAPKAPK5-AS1 high or low-group to explore the clinical significance of MAPKAPK5-AS1 in HCC. Statistical analysis found that high MAPKAPK5AS1 expression was significantly associated with large tumor size $(p=0.006)$, tumor multiplicity $(p=0.001)$, vascular invasion $(p=0.008)$ and advanced tumor-nodemetastasis (TNM) stages $(p=0.001)$ (Table 1$)$. Besides, TCGA data from UALCAN (http://ualcan.path.uab.edu/ index.html) revealed that high MAPKAPK5-AS1 level 

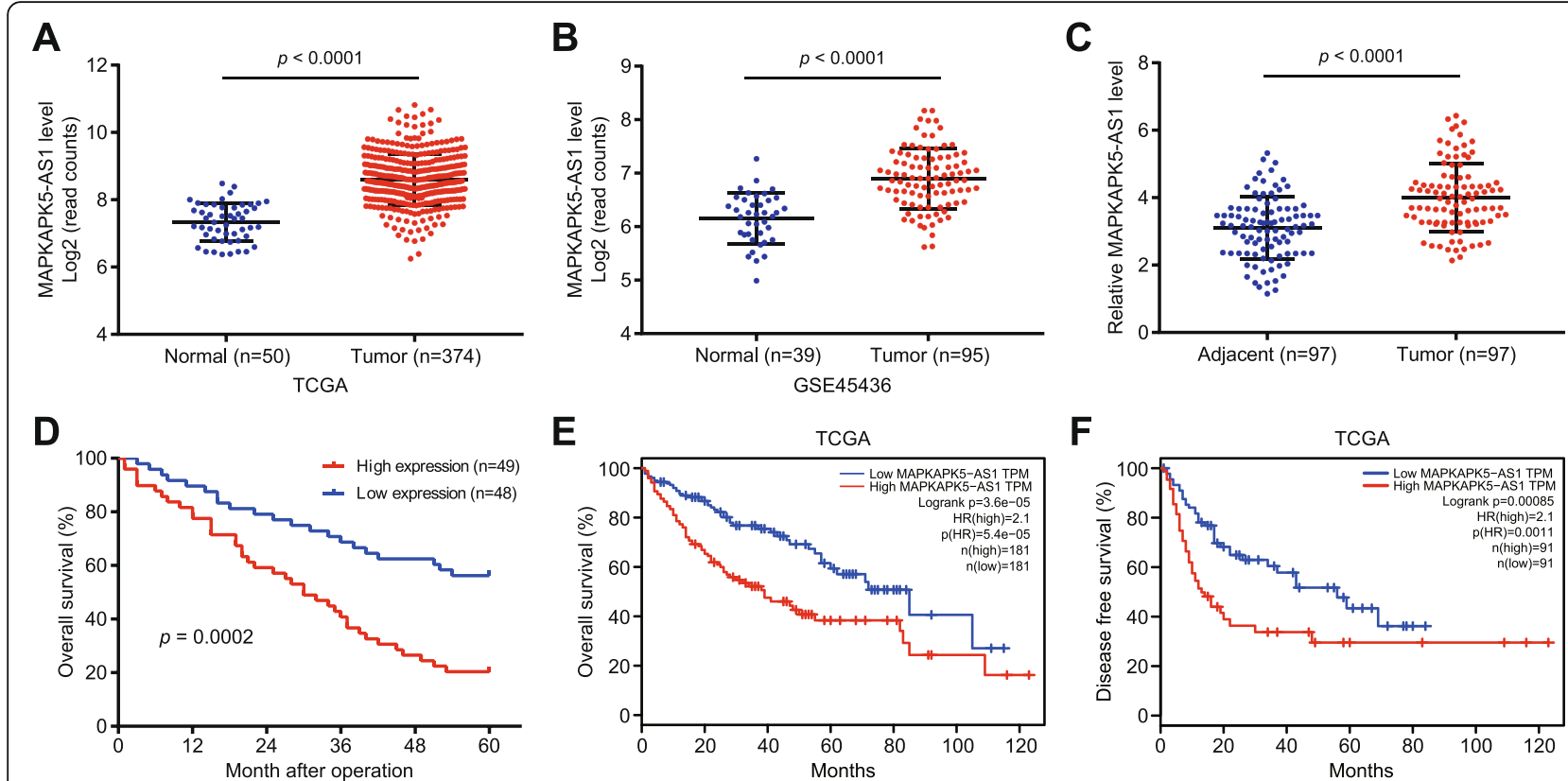

Fig. 1 MAPKAPK5-AS1 upregulation is associated with poor clinical features and prognosis in HCC. a TCGA dataset showed the high expression level of MAPKAPK5-AS1 in HCC tissues compared to normal tissues. b GSE45436 dataset further exhibited high expression level of MAPKAPK5-AS1 in HCC tissues. c The level of MAPKAPK5-AS1 in 97 pairs HCC tissues and adjacent non-tumor tissues. d Kaplan-Meier survival analysis showed that HCC patients with high MAPKAPK5-AS1 expression exhibited a worse overall survival. e-f TCGA data showed that high MAPKAPK5-AS1 expression indicated poor overall survival (OS) and disease-free survival (DFS)

had a positive association with the poor pathological stage and tumor grade of HCC patients (Additional File 3: Fig. S1C and S1D). Kaplan-Meier survival analysis for the $97 \mathrm{HCC}$ patients showed that HCC patients with high MAPKAPK5-AS1 expression exhibited a notably worse overall survival $(p=0.0002$, Fig. 1d). Moreover, TCGA data from GEPIA also showed that high MAPK APK5-AS1 expression indicated poor overall survival $\left(p=3.6 \times 10^{-5}\right.$, Fig. $\left.1 \mathrm{e}\right)$ and disease-free survival $(p=8.5 \times$ $10^{-4}$, Fig. 1f). Altogether, these results demonstrated MAPKAPK5-AS1 was upregulated in HCC, which was associated with poor clinical features and prognosis of $\mathrm{HCC}$ patients.

\section{MAPKAPK5-AS1 contributes to the proliferation and suppresses apoptosis of HCC cells in vitro}

To study the biological functions of MAPKAPK5-AS1, we stably knocked down MAPKAPK5-AS1 in HCCLM3 and MHCC97-H cells and overexpressed MAPKAPK5AS1 in Hep3B and Huh-7 cells. qRT-PCR results indicating the transfection efficiency of these four HCC cell lines with MAPKAPK5-AS1 overexpression or knockdown are exhibited in Additional File 4: Fig. S2. In MTT, colony formation, and EdU assays, MAPKAPK5-AS1 knockdown significantly inhibited viability and proliferation of HCCL M3 and MHCC97-H cells ( $p<0.05$, Fig. $2 \mathrm{a}, \mathrm{c}$, and e), whereas the viability and proliferation of Hep3B and Huh-
7 cells were markedly enhanced by MAPKAPK5-AS1 overexpression $(p<0.05$, Fig. $2 \mathrm{~b}, \mathrm{~d}$, and $\mathrm{f})$. Besides, flow cytometry analysis demonstrated that MAPKAPK5-AS1 deficiency in HCCLM3 and MHCC97-H cells obviously induced cell apoptosis $(p<0.05$, Fig. $2 \mathrm{~g}$ ), while cell apoptosis was strongly suppressed by MAPKAPK5-AS1 overexpression in Hep3B and Huh-7 cells ( $p<0.05$, Fig. $2 \mathrm{~h}$ ). These results above indicated that MAPKAPK5-AS1 promoted the proliferation of HCC cells by suppressing cell apoptosis.

\section{MAPKAPK5-AS1 facilitates the mobility and EMT process of HCC cells in vitro}

Next, transwell migration and invasion assays were carried out to explore the effect of MAPKAPK5-AS1 on cells mobility, and the results showed that MAPKAPK5AS1 knockdown in HCCLM3 and MHCC97-H remarkably curbed the migration and invasion of tumor cells $(p<0.05$, Fig. 3a), whereas the mobility of Hep3B and Huh-7 cells was notably enhanced by overexpressing MAPKAPK5-AS1 $(p<0.05$, Fig. 3b). Considering that EMT is a curial mechanism mediating cancer metastasis, the effect of MAPKAPK5-AS1 on the EMT process of tumor cells was further investigated. Western blotting assays showed that MAPKAPK5-AS1 knockdown in HCCLM3 and MHCC97-H substantially increased the expression of epithelial markers (E-cadherin) and 


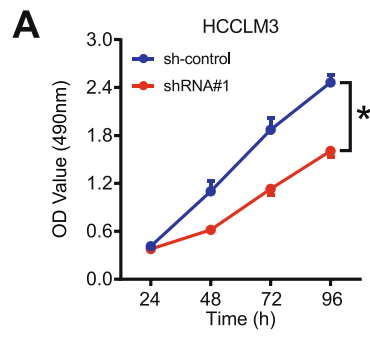

C
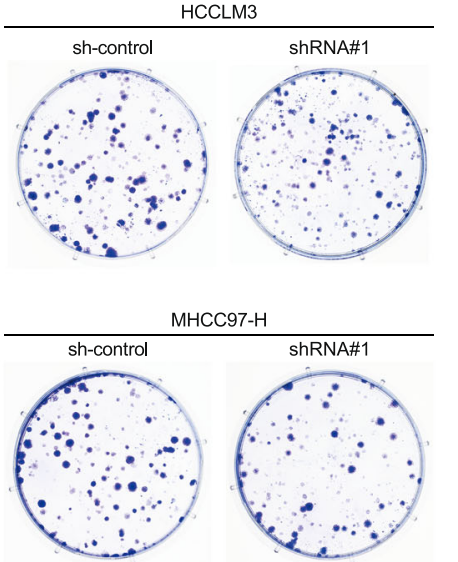

$\mathbf{E}$
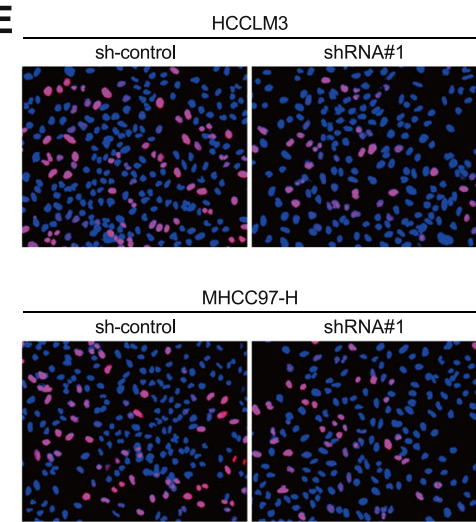

G
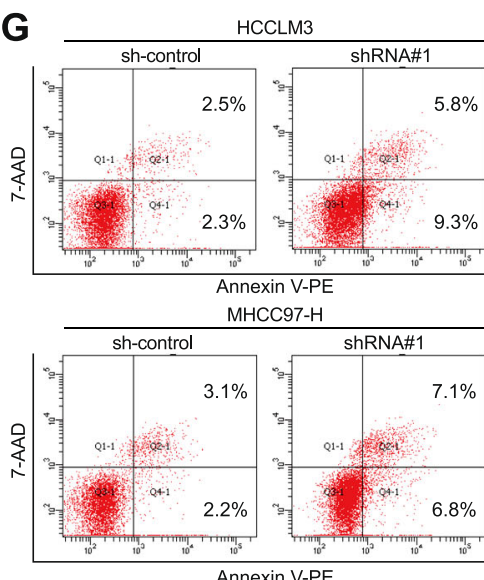

Annexin V-PE
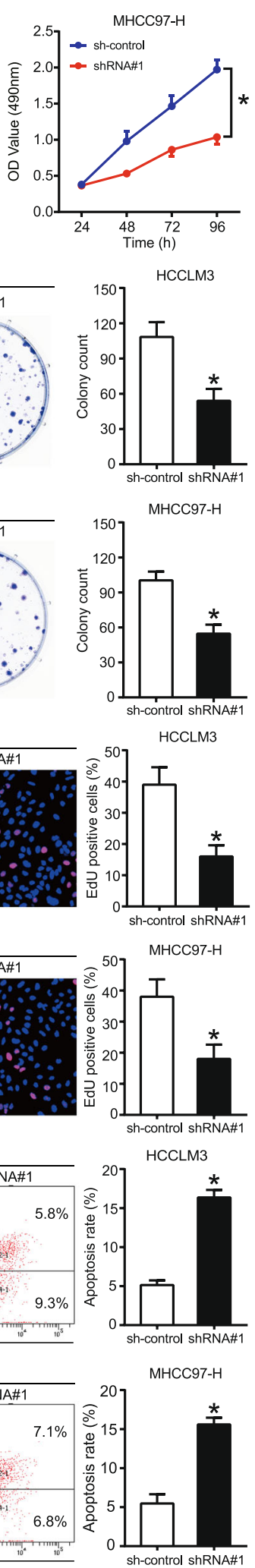

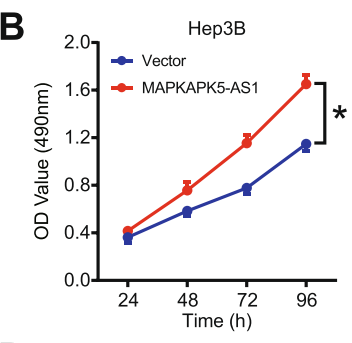

D

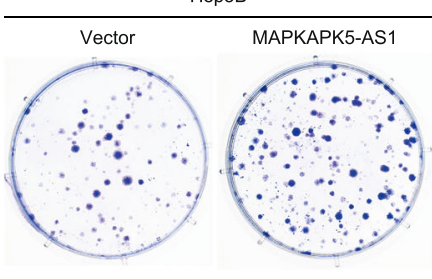

Huh-7
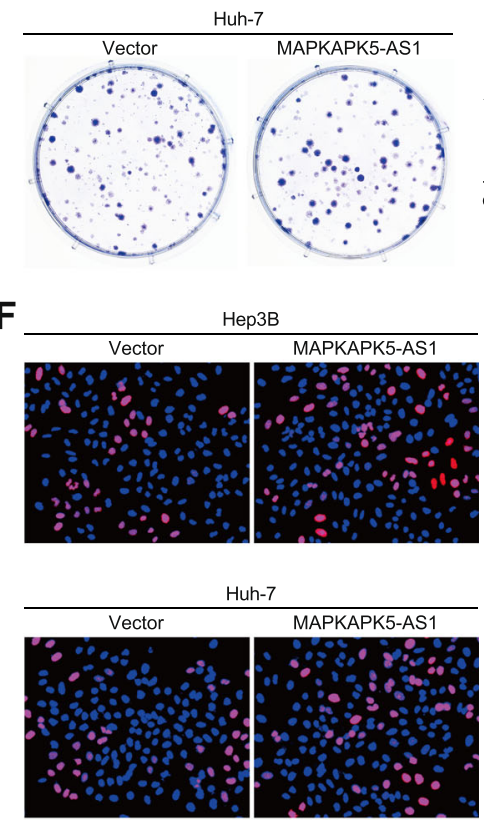

$\mathbf{H}$
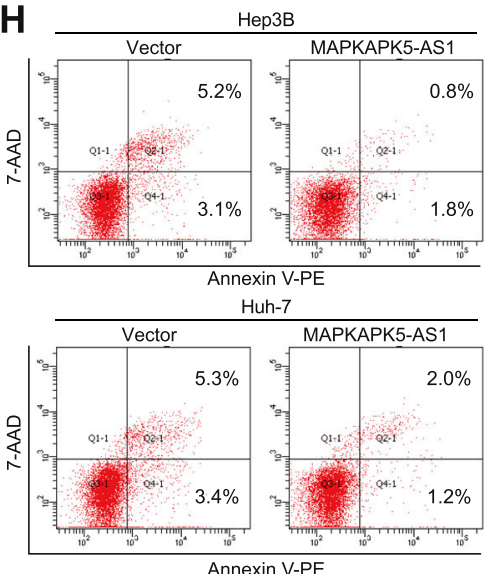

Huh-7

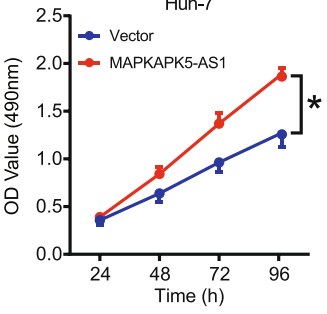

Нер3В

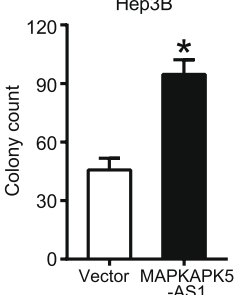

Huh-7 $^{-A S}$

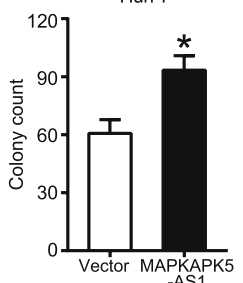

Hep3B

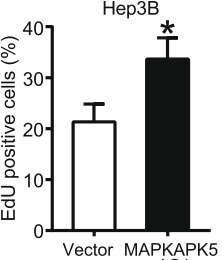

Huh-7 $^{- \text {AS1 }}$
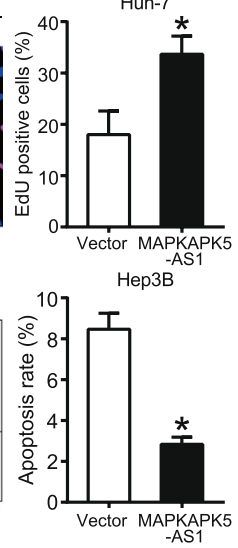

Huh-7

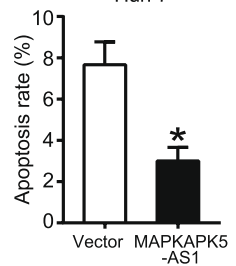

Fig. 2 (See legend on next page.) 
(See figure on previous page.)

Fig. 2 MAPKAPK5-AS1 promotes proliferation and inhibits apoptosis of HCC cells. $\mathbf{a}-\mathbf{b}$ MTT assay was carried out to examined the effect of MAPK APK5-AS1 knockdown or overexpression on HCC cells viability. c-d Colony formation confirmed the negative regulatory effect of MAPKAPK5-AS1 on tumor cells proliferation. e-f The influence of MAPKAPK5-AS1 on DNA synthesis activity of HCC cell was evaluated by EdU assay. $\mathbf{g}$ - $\mathbf{h}$ The effect of MAPKAPK5-AS1 on cell apoptosis was assessed by flow cytometry. ${ }^{*} p<0.05$

decreased the level of the mesenchymal markers (N-cadherin and Vimentin) (Fig. 3c). The opposite regulatory effect on EMT markers expression was observed after overexpressing MAPKAPK5-AS1 in Hep3B and Huh-7 cells (Fig. 3d). Thus, our above results demonstrated that MAPKAPK5-AS1 contributed to HCC cells' mobility by regulating the EMT process.

\section{MAPKAPK5-AS1 promotes the growth and metastasis of HCC cells in vivo}

These above mentioned in vitro experimental results indicate that MAPKAPK5-AS1 functions as an oncogene in HCC progression. To make the conclusion more credible, we performed various in vivo experiments to investigate the effects of MAPKAPK5-AS1 on the growth and metastasis of HCC cells. HCCLM3 cells with MAPKAPK5-AS1 knockdown or Hep3B cells with MAPKAPK5-AS1 overexpression were implanted into nude mice via subcutaneous injection or tail vein injection. The results indicated that knocking down MAPKAPK5-AS1 in HCCLM3 cells remarkably suppressed tumor cells' growth and lung metastasis in vivo compared with the control group $(p<0.05$, Fig. 4a and c). By contrast, overexpressing MAPKAPK5AS1 in Hep3B cells significantly promoted tumor cells' growth and lung metastasis in vivo $(p<0.05$, Fig. $4 \mathrm{~b}$ and d). Subsequently, RNA in subcutaneous tumors was
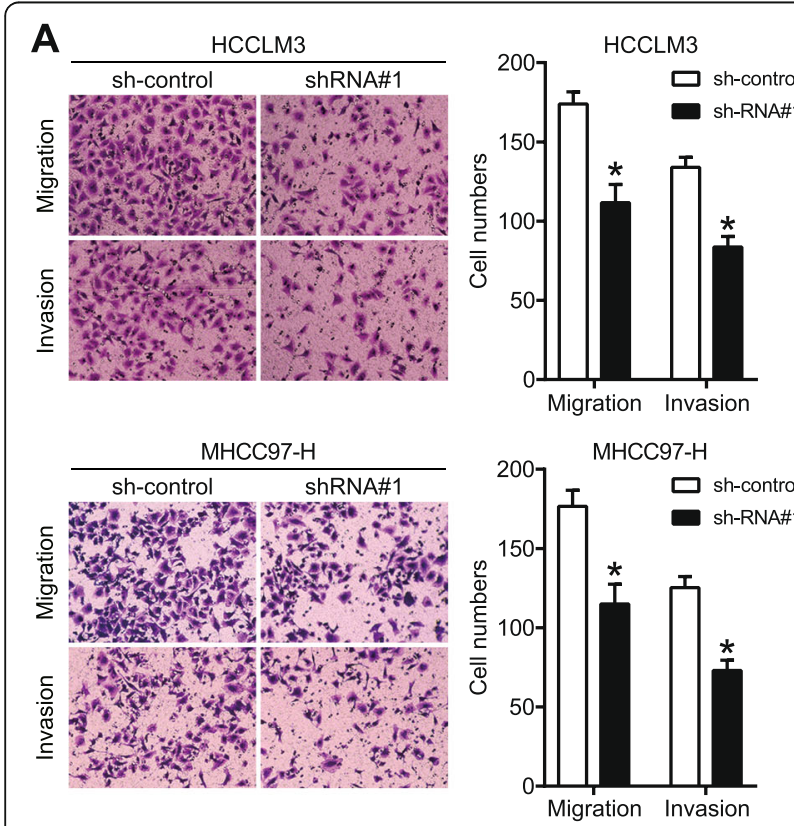

C
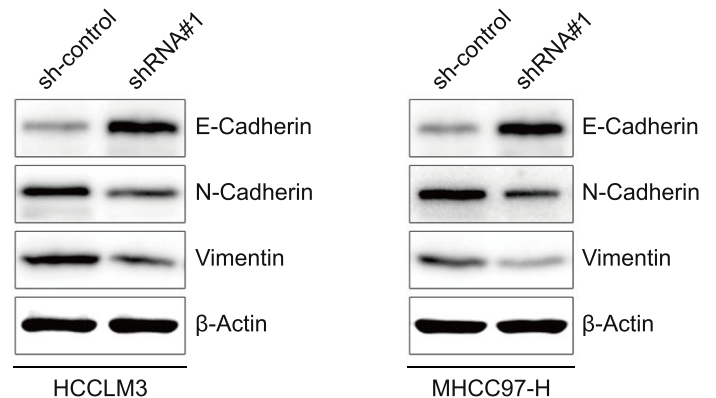
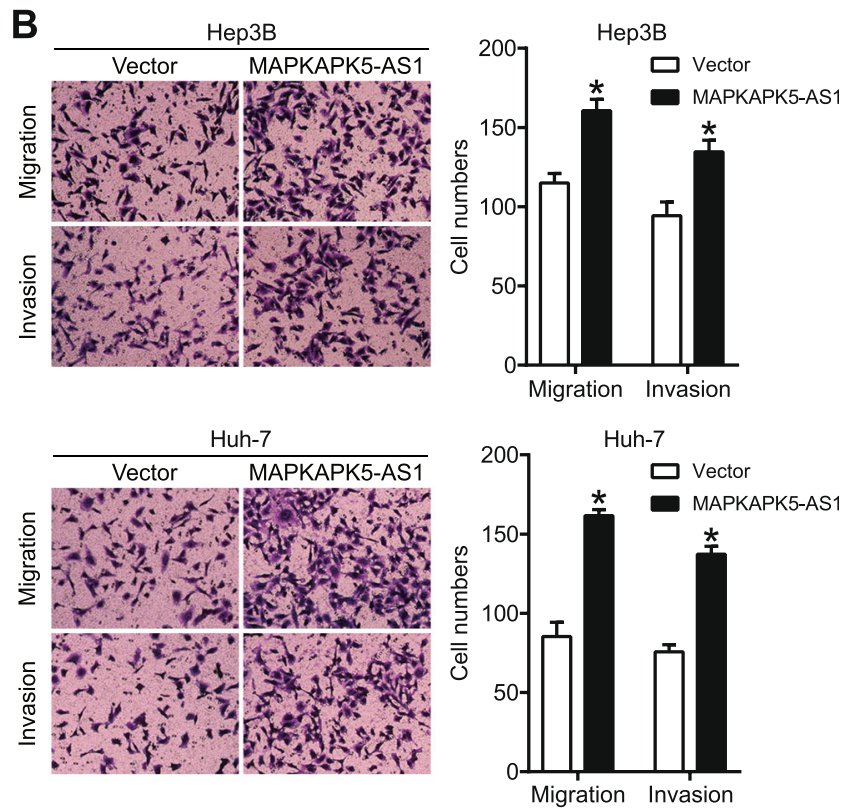

D
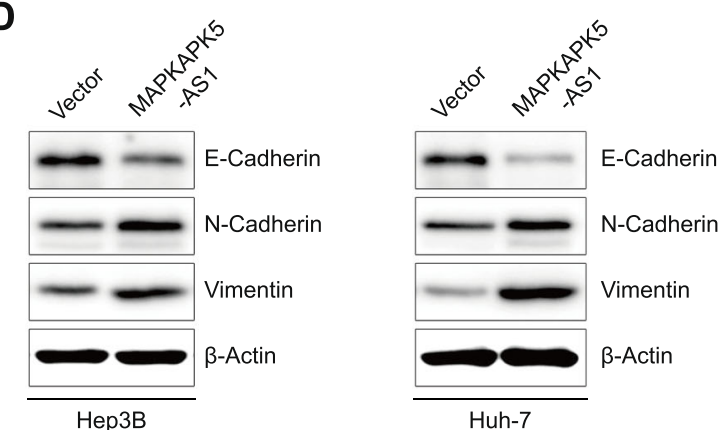

Fig. 3 MAPKAPK5-AS1 enhances migration, invasion, and EMT process of HCC cells. a-b Transwell assay were performed to evaluate the effects of MAPKAPK5-AS1 on the tumor cells abilities of migration and invasion. c-d Expression of EMT markers was detected by western blotting to assess the effect of MAPKAPK5-AS1 on EMT process. ${ }^{*} p<0.05$ 
A
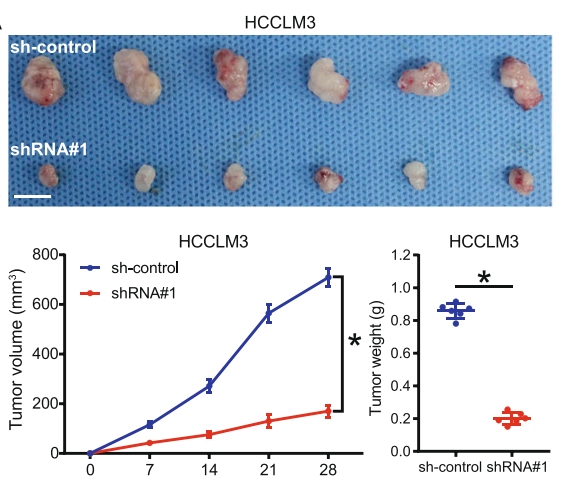

C

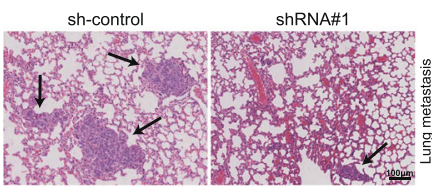

E

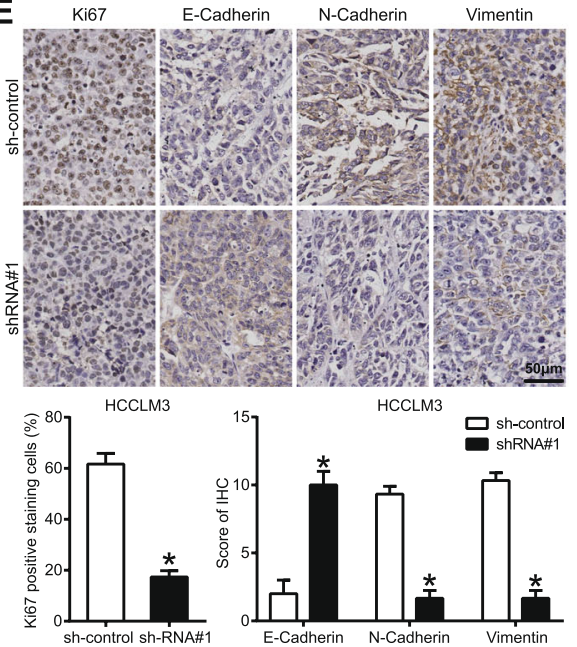

B
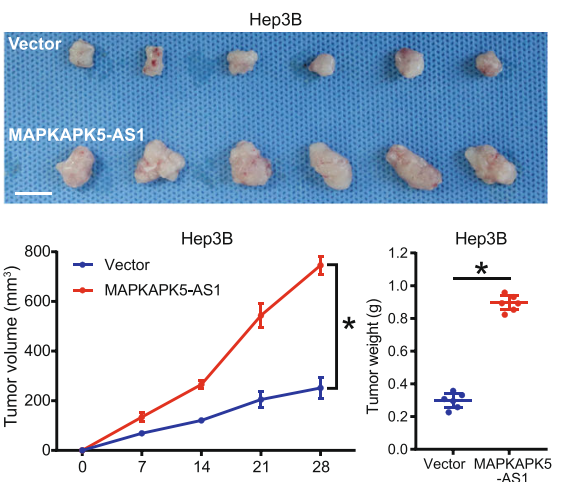

HCCLM3 D

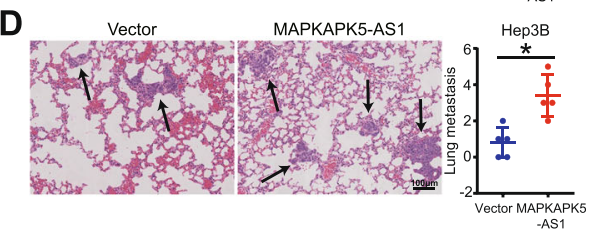

$\mathbf{F}$
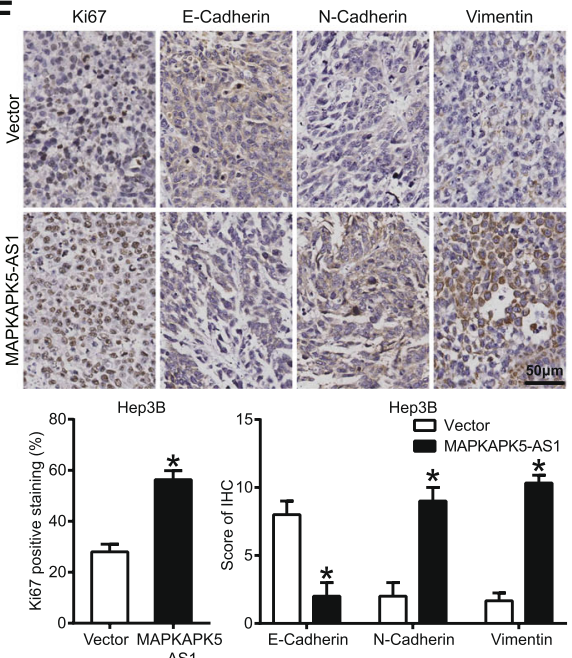

Fig. 4 MAPKAPK5-AS1 promotes the growth and metastasis of HCC cell in vivo. a-b Xenograft tumor model showed the negative regulatory effect of MAPKAPK5-AS1 on tumor size, growth rate, and tumor weight. $\mathbf{c}-\mathbf{d}$ Representative images of tail vein injection model and the number of lung metastatic nodes. e-f $1 \mathrm{HC}$ results of Ki67 and EMT markers for xenograft tumors. ${ }^{*} p<0.05$

extracted to detect the expression of MAPKAPK5-AS1 by RT-qPCR assay, and the results showed that the level of MAPKAPK5-AS1 in tumor tissues arising from the MAPKAPK5-AS1 knockdown group was significantly lower than that in the control group $(p<0.05$, Additional File 5: Fig. S3A), and the level of MAPKAPK5-AS1 in tumor tissues arising from MAPKAPK5-AS1 overexpression group was significantly higher than that in the control group $(p<0.05$, Additional File 5: Fig. S3B). To further validate the influence of MAPKAPK5-AS1 on HCC cell growth and EMT phenotype, we performed IHC analysis for tumors formed by different HCC cells. Results showed that knocking down MAPKAPK5-AS1 significantly decreased the positive rate of Ki67 staining, increased the expression of E-cadherin, and decreased the level of $\mathrm{N}$-cadherin and vimentin in tumors formed by
HCCLM3 cells $(p<0.05$, Fig. 4e). By contrast, overexpressing MAPKAPK5-AS1 in Hep3B cells promoted the growth and EMT progression $(p<0.05$, Fig. $4 \mathrm{f})$. Together, these results confirmed that MAPKAPK5-AS1 promoted $\mathrm{HCC}$ cell growth and metastasis in vivo.

\section{MAPKAPK5-AS1 sponges miR-154-5p in HCC cells}

LncRNAs regulate gene expression at the transcriptional level or post-transcriptional level, depending on lncRNAs subcellular localization. A subcellular fractionation assay was applied to investigate the molecular mechanism involved in the MAPKAPK5-AS1 function. Results indicated that MAPKAPK5-AS1 was mainly located in the cytoplasm of HCC cells (Fig. 5a and Additional File 6: Fig. S4A), which suggested that MAPKAPK5-AS1 may exert its biological function through its mechanism of competitive endogenous 

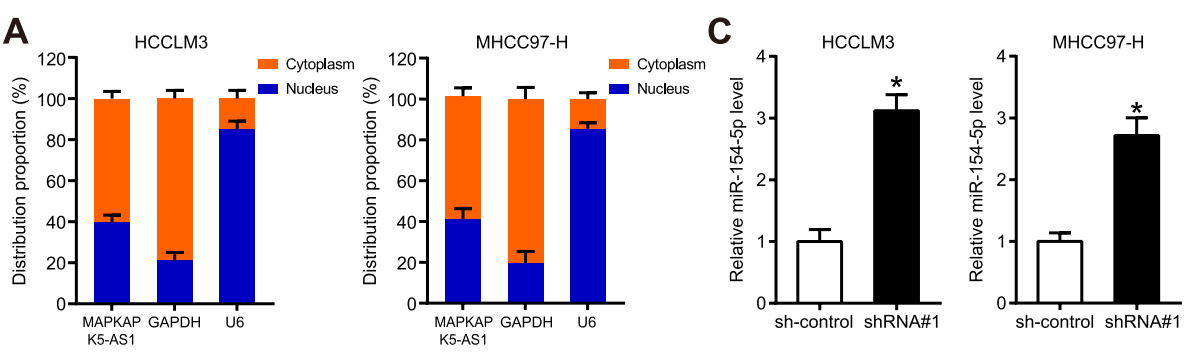

B
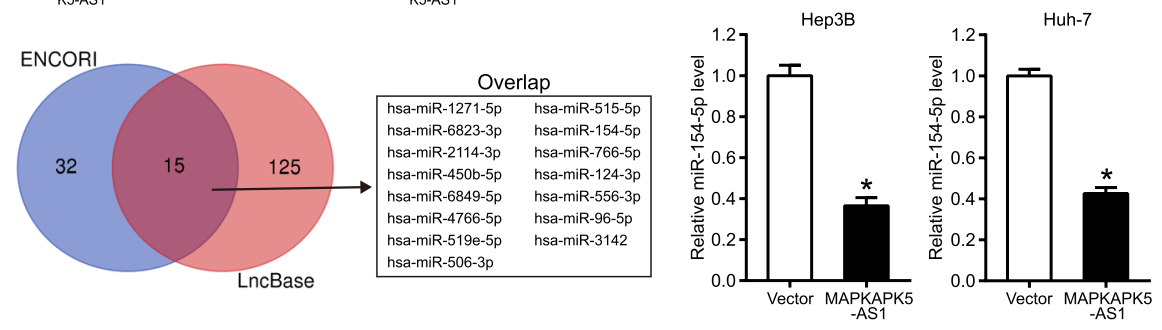

D
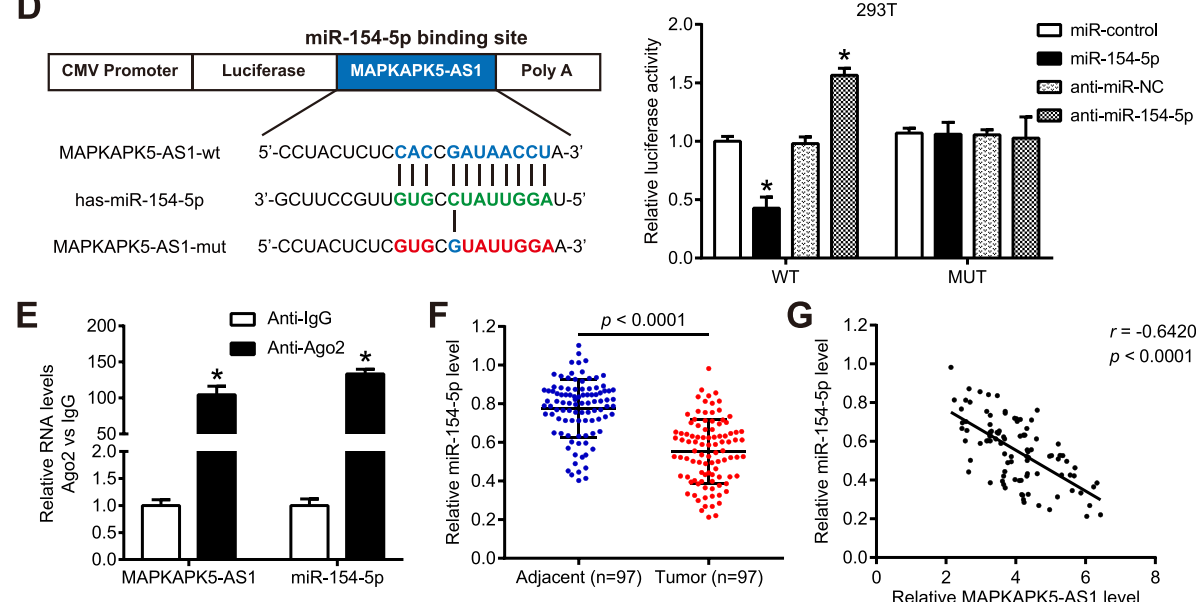

Fig. 5 MAPKAPK5-AS1 acts as a sponge of miR-154-5p in HCC cells. a Subcellular fractionation assay was applied to determine MAPKAPK5-AS1 subcellular localization. b 15 putative targets of MAPKAPK5-AS1 was predicted by applying two bioinformatics prediction tools. C QRT-PCR showed that MAPKAPK5-AS1 negatively regulated miR-154-5p expression in HCC cells. $\mathbf{d}$ The predicted binding sites between miR-154-5p and MAPKAPK5-AS1 and mutant sequences of the potential miR-154-5p binding site in MAPKAPK5-AS1. Additionally, the result of luciferase reporter assay is shown here. (E) Anti-Ago2 RIP assay was performed in HCCLM3 with miR-154-5p overexpression, followed by qRT-PCR to detect the level of MAPKAPK5-AS1 or miR-154-5p associated with Ago2. $\mathbf{f}$ The expression level of miR-154-5p in HCC tissues and adjacent non-tumor tissues. $\mathbf{g}$ The relativity between MAPKAPK5-AS1 and miR-154-5p in HCC tissues. ${ }^{*} p<0.05$

RNA (ceRNA). Then, ENCORI and LncBase, these two online bioinformatics websites, were used to predict potential targeted miRNAs for MAPKAPK5-AS1. Fifteen miRNAs were overlapping between these two predicted lists among these predicted miRNAs (Fig. 5b). Previous studies showed that only miR-1271-5p [25, 26], miR-154-5p [27-29], and miR-450b-5p [30, 31], these three miRNAs, were downregulated and acted as tumor suppressor genes. To determine which miRNA can actually be regulated by MAPK APK5-AS1, we detected these three miRNAs expression levels respectively after knocking down MAPKAPK5-AS1 in HCC cells. Our data revealed that only miR-154-5p could be significantly negatively regulated by MAPKAPK5-AS1 in HCC cells $(p<0.05$, Additional File 6: Fig. S4B; $p<0.05$, Fig. $5 c)$. Next, Ago2-RIP and luciferase reporter assays were performed to identify whether there was direct interaction between MAPKAPK5-AS1 and miR-154-5p. Firstly, the Ago2-RIP assay showed that the Ago2 antibody could pull down both endogenous MAPKAPK5-AS1 and miR-154-5p, which confirmed that MAPKAPK5-AS1 and miR-154-5p could bind with Ago2 in RISC ( $p<0.05$, Fig. 5e). Secondly, luciferase reporter assay showed that miR-154-5p could significantly negatively regulate the luciferase activity of vectors containing wild-type MAPKAPK5-AS1 but failed to reduce that of the mutant vector $(p<0.05$, Fig. $5 \mathrm{~d})$, which demonstrated that miR-154-5p was directly bound to MAPKAPK5AS1. Subsequently, the level of miR-154-5p in paired tissue samples was detected. As expected, miR-154-5p was downregulated in HCC tissue $(p<0.0001$, Fig. $5 \mathrm{f}$ and Additional File 6: Fig. S4C) and exhibited a negative correlation with the 
expression of MAPKAPK5-AS1 $(r=-0.6420, p<0.0001$, Fig. $5 \mathrm{~g})$. These results illustrated that MAPKAPK5-AS1 acted as a molecular sponge for miR-154-5p in HCC cells.

\section{MiR-154-5p mediates the biological function of MAPK APK5-AS1}

Recuse assays were conducted to explore whether miR154-5p mediated the biological function of MAPKAPK5AS1. The results of MTT, colony formation, and EdU assays revealed that miR-154-5p inhibitor partially reversed the anti-proliferation effect of sh-MAPKAPK5AS1 ( $p<0.05$, Fig. 6a-c), while miR-154-5p mimics could impair the pro-proliferation effect of MAPKAPK5AS1 $(p<0.05$, Additional File 7: Fig. S5A-C). Additionally, flow cytometry analysis results showed that cell apoptosis induced by MAPKAPK5-AS1 knockdown was inhibited by co-transfecting with miR-154-5p inhibitor $(p<0.05$, Fig. 6d) while overexpressing miR-154-5p induced cell apoptosis inhibited by MAPKAPK5-AS1 $(p<$ 0.05, Additional File 7: Fig. S5D). Subsequently, transwell migration and invasion assays exhibited that the motility decreased by knocking down MAPKAPK5-AS1 was driven by inhibiting miR-154-5p expression in HCCLM3 cells $(p<0.05$, Fig. $6 \mathrm{e})$, whereas the motility enhanced by MAPKAPK5-AS1 overexpression was impaired by miR154-5p mimics in Hep3B cells $(p<0.05$, Additional File 7: Fig. S5E). Similarly, western blot assay confirmed that the EMT progression hindered by MAPKAPK5-AS1 knockdown was partially recovered by a miR-154-5p inhibitor $(p<0.05$, Fig. $6 \mathrm{f})$, whereas the EMT progression promoted by MAPKAPK5-AS1 was partially abolished by miR-154-5p mimics $(p<0.05$, Additional File 7: Fig. S5F). These recuse assays confirmed that MAPKAPK5AS1 exerted its biological function through sponging miR-154-5p in HCC cells.

\section{PLAGL2 is the direct target of miR-154-5p}

Considering that miRNAs exert its function by modulating the expression of target genes, miRDB, PicTar, and TargetScan, these three bioinformatics databases, were used to

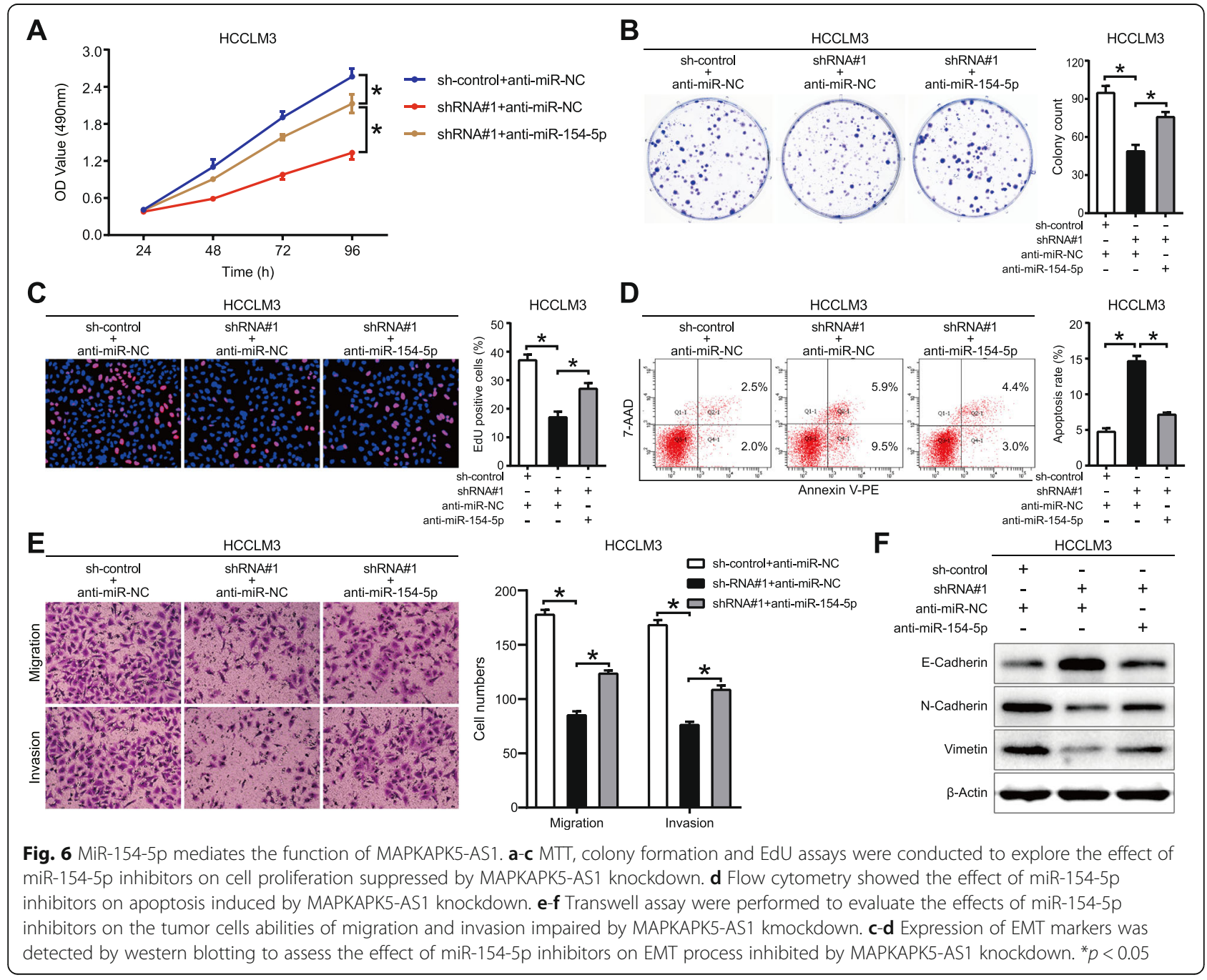


predict the potential targets of miR-154-5p. As shown in Fig. $7 \mathrm{a}$, ten targets were overlapping in the prediction results of these three databases. Among these genes, only DYRK1A [32], VPS4B [33], PLAGL2 [34], these three genes have been reported to act as tumor-promoter in HCC. Then, we detected the three mRNAs' levels in HCCLM3 cells transfected with miR-154-5p mimics, and we found that just PLAGL2 mRNA could be significantly negatively altered ( $p<0.05$ Fig. $7 b)$. Subsequently, we found that miR154-5p mimics significantly decreased PLAGL2 mRNA and protein levels in both HCCLM3 and MHCC97-H cells $(p<$ 0.05, Additional File 8: Fig. S6A and Fig. 7c). Also, PLAGL2 mRNA and protein expression were elevated by miR-154$5 p$ inhibitor in both Hep3B and HuH-7 cells $(p<0.05$, Additional File 8: Fig. S6B and Fig. 7c). These data confirmed that PLAGL2 expression could be negatively regulated by miR-154-5p in HCC cells. Next, we attempted to explore whether there exists direct binding between miR-154-5p and PLAGL2 mRNA. The putative binding sequence was listed in Fig. 7d. Further luciferase reporter assay showed that miR-154-5p mimics or inhibitors could remarkably negatively modulate the luciferase activity of wild-type PLAGL2 vector but not the mutant vector $(p<0.05$, Fig. 7d). Furthermore, we found that MAPKAPK5-AS1 could partially abolish the inhibitory effect of miR-154-5p on PLAGL2 protein expression in both HCCLM3 and MHCC97-H cells, and miR-154-5p also could partially reverse the promotional effect of MAPKAPK5-AS1 on PLAG L2 protein expression in both Hep3B and HuH-7 cells (Fig. 7e). Moreover, we observed that PLAGL2 level was elevated in HCC tissues compared with adjacent non-tumor tissues $(p<0.0001$, Fig. $7 \mathrm{f}$ and Additional File 8: Fig. S6C). Additionally, statistical analysis showed that the expression of PLAGL2 was negatively correlated with miR-154-5p expression $(r=-0.6889, p<0.0001$, Fig. $7 \mathrm{~g})$, while positively correlated with MAPKAPK5-AS1 expression $(r=$ $0.6655, p<0.0001$, Fig. 7h; $r=0.294, p<0.0001$, Additional File 8: Fig. S6D). Finally, the PLAGL2 expression in xenograft tumors was detected by western blotting, and the results showed that MAPKAPK5-AS1 could positively modulate the expression of PLAGL2 in xenograft tumors (Additional File 8: Fig. S6E). Therefore, our data indicated that MAPKAPK5-AS1 positively altered PLAGL2 expression by inhibition of miR-154-5p.

\section{PLAGL2/EGFR/AKT pathway mediates the tumor- promoting effect of MAPKAPK5-AS1}

Given that previous research has confirmed that PLAGL2 functions as a transcriptional regulator of EGFR and promotes HCC cell proliferation, migration, and invasion through the EGFR-AKT pathway [34], we have determined that MAPKAPK5-AS1 upregulates PLAGL2 level by sponging miR-154-5p. Recuse assays were conducted to investigate whether PLAGL2/EGFR/AKT mediated the biological function of MAPKAPK5-AS1. The results of MTT, colony formation, and EdU assays revealed that restriction of MAPKAPK5-AS1 knockdown on cell proliferation was partially repealed by PLAGL2 overexpression in HCCLM3 $(p<0.05$, Fig. 8a-c), and PLAGL2 knockdown reversed the promoting effect of MAPKAPK5-AS1 on proliferation $(p<0.05$, Additional File 9: Fig. S7A-C). In addition, we found that MAPKAPK5-AS1 knockdown induced HCCLM3 cell apoptosis, which could be partially abolished by PLAGL2 overexpression ( $p<0.05$, Fig. $8 d$ ). Similarly, knocking down PLAGL2 in Hep3B cells attenuated the inhibitory effect of MAPKAPK5-AS1 on cell apoptosis $(p<0.05$, Additional File 9: Fig. S7D). Subsequently, results of transwell and western blot assays indicated that the motility and EMT of HCCLM3 weakened by MAPKAPK5-AS1 knockdown was enhanced by PLAG L2 ( $p<0.05$, Fig. 8e and $\mathrm{f}$ ), and the motility and EMT of Hep3B enhanced by MAPKAPK5-AS1 were weakened by PLAGL2 knockdown $(p<0.05$, Additional File 9: Fig. S7E and 7F). Moreover, we also found that silencing MAPK APK5-AS1 inactivated epidermal growth factor receptor (EGFR)/AKT signaling in HCCLM3 cells, which could be reversed by PLAGL2 (Fig. 8f), and vice versa in Hep3B cells (Additional File 9: Fig. S7F). In addition, a series of experiments were performed to detect the effect of MK2206, the inhibitor of AKT, on the tumor-promoting effect of MAPKAPK5-AS1, and the results showed that MAPKAPK5-AS1 overexpression enhanced the proliferation, the motility and EMT of Hep3B, and reduced Hep3B cell apoptosis, which could be partially reserved by MK2206 treatment (Additional File 10: Fig. S8). Overall, these recuse experiments demonstrated that PLAGL2/ EGFR/AKT pathway mediated the biological function of MAP-KAPK5-AS1 in HCC cells.

\section{Hypoxia regulates a MAPKAPK5-AS1/PLAGL2/HIF-1a signaling loop}

Considering that PLAGL2 has been reported to be a hypoxia-responsive gene, we investigated whether hypoxia was involved in MAPKAPK5-AS1 expression regulation based on our above research results. Our lncRNA microarray data (GSE155505) showed that the MAPKAPK5-AS1 level was elevated under hypoxic conditions (Fig. 9a), and this finding was supported by qRT-PCR ( $p<0.05$, Fig. 9b). In addition, western blotting assays exhibited that hypoxia induced the upregulation of PLAGL2 (Fig. 9b), which was consistent with the previous study [34]. Furthermore, we found that HIF-1 $\alpha$ knockdown significantly impaired the upregulation of MAPKAPK5-AS1 and PLAGL2 induced by hypoxia $(p<0.05$, Fig. 9b), which indicated that HIF-1 $\alpha$ mediated the regulation of hypoxia on the expression of MAPKAPK5-AS1 and PLAGL2. Next, JASPAR webtool predicted a potential hypoxia-response element (HRE) in the promoter of MAPKAPK5-AS1 (Fig. 9c). Consequently, 


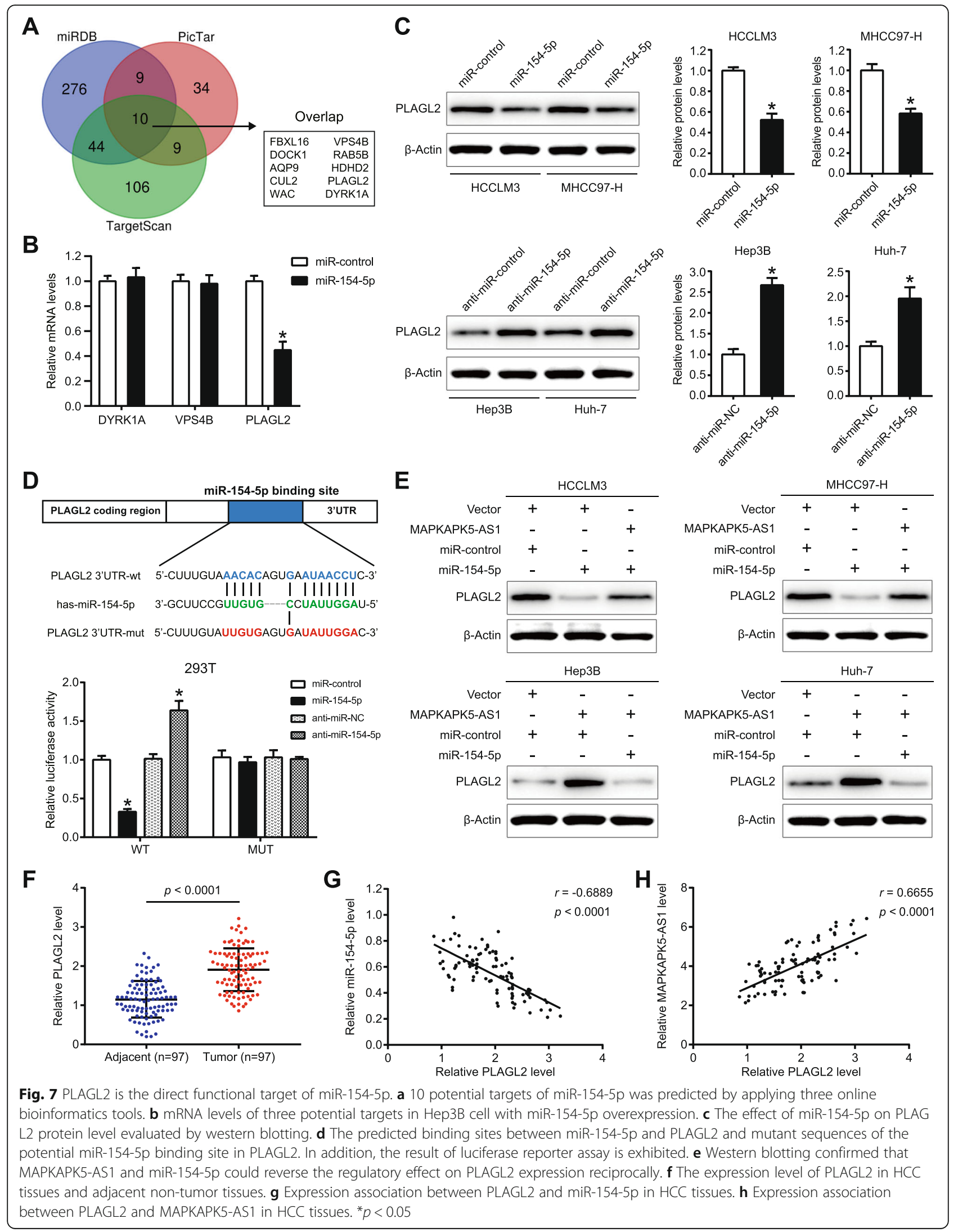


we established a luciferase report vector containing the MAPKAPK5-AS1 promoter. And the results showed that hypoxia remarkably enhanced the luciferase activity in cells transfected with the vector containing MAPKAPK5-AS1 promoter, and HIF-1 $\alpha$ knockdown could reverse the luciferase activity induced by hypoxia, which suggested MAPK APK5-AS1 promoter containing the functional HRE $(p<$
0.05, Fig. 9d). Moreover, the ChIP assay further confirmed the direct binding between HIF- $1 \alpha$ and HRE in the MAPK APK5-AS1 promoter $(p<0.05$, Fig. 9e). Then the regulation of MAPKAPK5-AS1 on HIF-1 $\alpha$ and PLAGL2 was studied. Western blotting assays revealed that the upregulation of HIF- $1 \alpha$ and PLAGL2 induced by hypoxia was obviously attenuated by MAPKAPK5-AS1 knockdown $(p<0.05$, Fig.

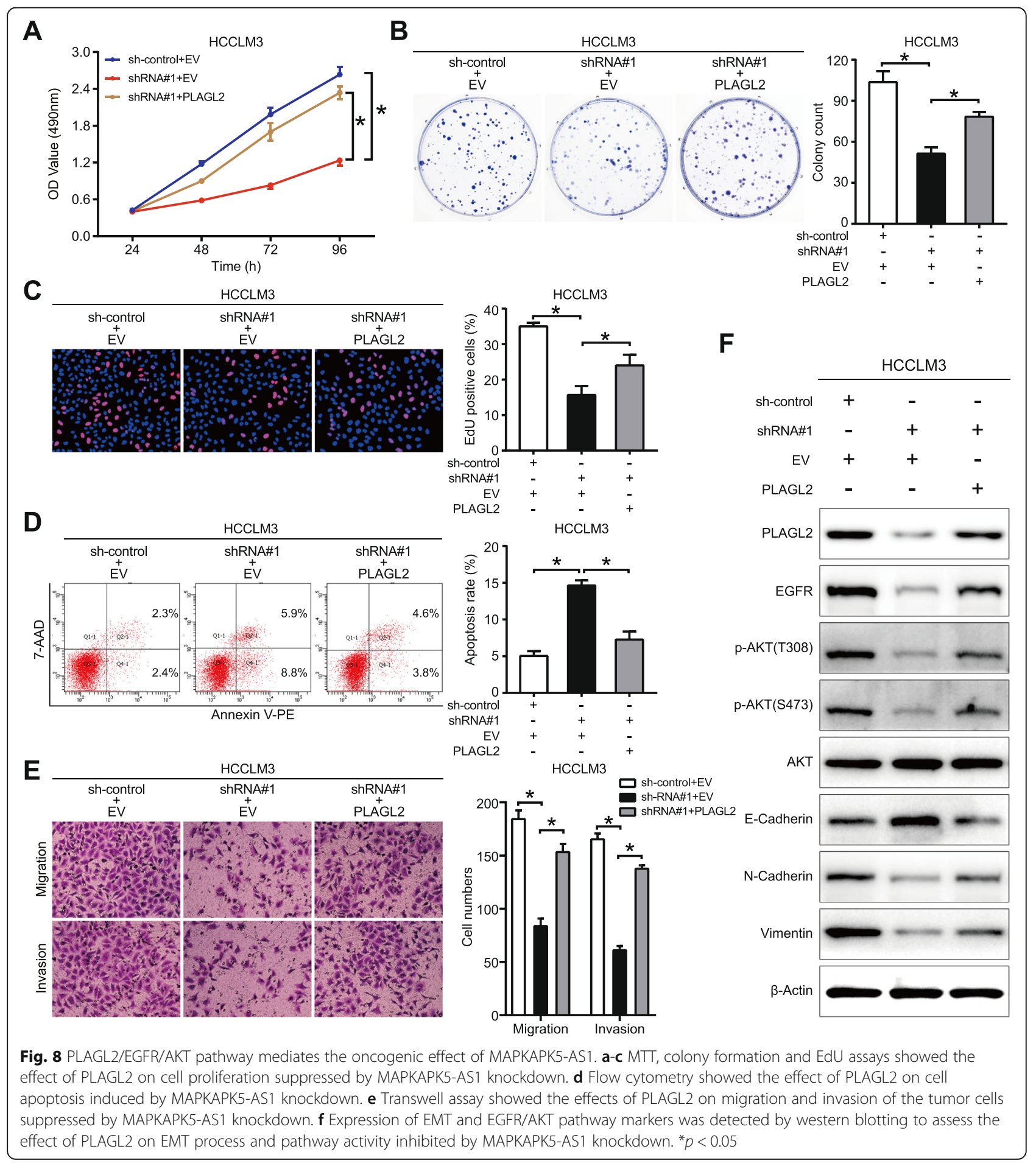


9f). Finally, we explored the effect of PLAGL2 on HIF-1 $\alpha$ and MAPKAPK5-AS1. The results showed that hypoxia elevated the level of HIF- $1 \alpha$ and MAPKAPK5-AS1, which could be partly reversed by PLAGL2 knockdown $(p<0.05$, Fig. 9g). Our results collectively indicated that HIF- $1 \alpha$ could directly bind to the promoter to activate MAPKAPK5-AS1 transcription, and MAPKAPK5-AS1 regulated HIF- $1 \alpha$ expression through PLAGL2 form a hypoxia-mediated MAPKAPK5-AS1/PLAGL2/HIF-1 $\alpha$ signaling loop in HCC.

\section{Discussion}

Recently, increasing lncRNAs involved in hepatocellular carcinoma progression have been reported [35-37], which provides us with a new perspective to understand tumor occurrence and development, thereby offering us new targets for treating hepatocellular carcinoma. LncRNA MAPKAPK5-AS1 is identified as a novel critical oncogene in colorectal cancer [38]. However, the role of MAPKAPK5-AS1 in HCC has not been investigated. Here, we showed that lncRNA MAPKAPK5-AS1 expression was elevated in $\mathrm{HCC}$, and its high expression was significantly positively associated with poor clinical features and prognosis of hepatocellular carcinoma patients. Functionally, MAPKAPK5-AS1 acts as a tumorpromotor through contributing to HCC cell growth and metastasis in vitro and in vivo. Our study identified the oncogenic role of MAPKAPK5-AS1 for the first time in hepatocellular carcinoma.

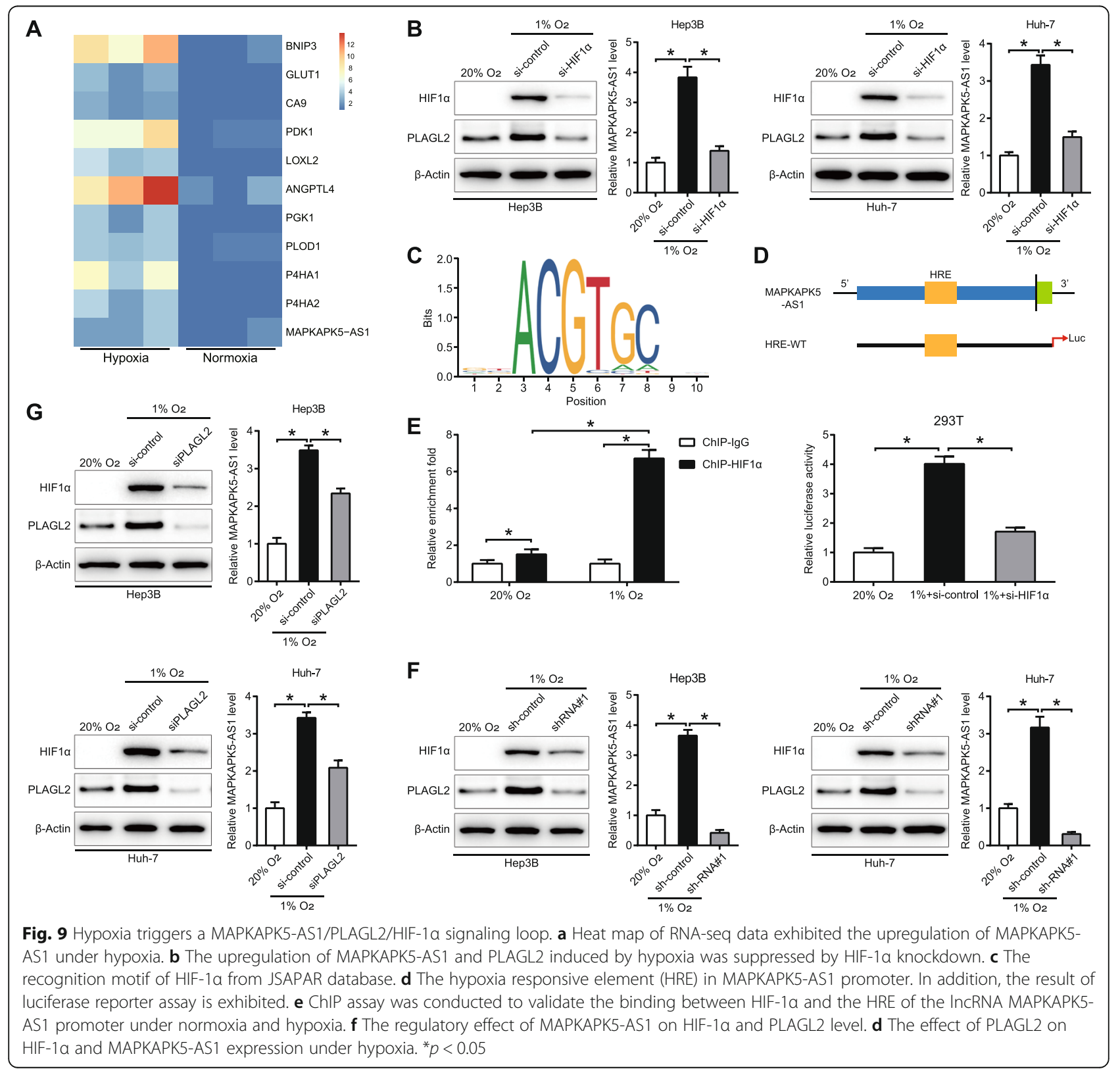




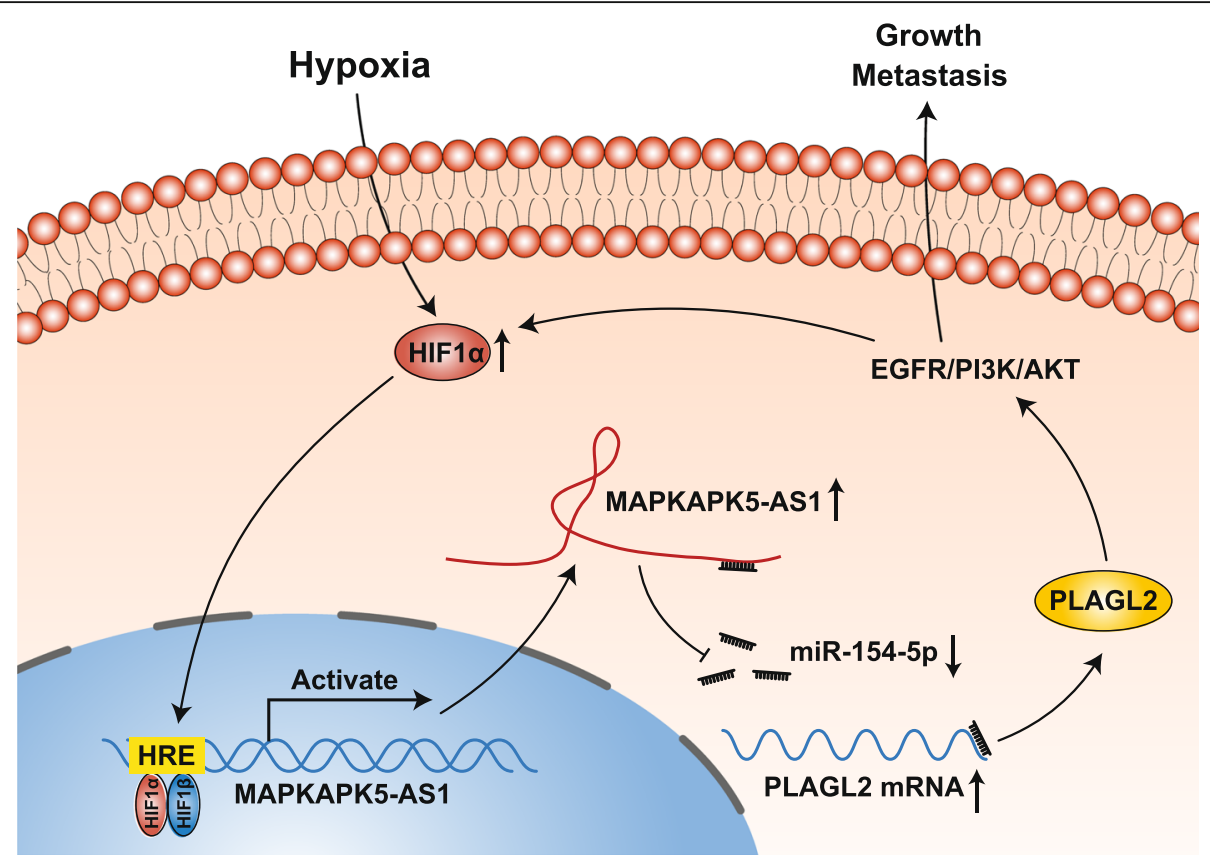

Fig. 10 Schematic of the findings of the present study

The subcellular localization of lncRNAs can indicate the mechanism mediating their biological functions. Studies have shown that IncRNAs located in the cell nucleus mainly regulate gene expression through binding with various proteins. In contrast, IncRNAs, which are located in the cytoplasm, generally regulate gene expression at the post-transcriptional level through the ceRNA mechanism [39]. In the study, we observed that MAPKAPK5-AS1 was mainly located in the cytoplasm by subcellular fractionation assay, which suggested that MAPKAPK5-AS1 might exert its oncogenic role in $\mathrm{HCC}$ cells through functioning as a miRNA sponge. Therefore, we next predicted the potential target of MAPKAPK5-AS1, miR-154-5p, by online bioinformatics website, which was further verified through luciferase reporter assay and Ago2-RIP assay. In addition, we found that there was a negative correlation between miR-154-5p and MAPKAPK5-AS1 expression in HCC tissue. Significantly, we performed a series of recusing assays, which demonstrated that miR-154-5p mediated the oncogenic function of MAPKAPK5-AS1 in HCC cells. In summary, we determined that MAPKAPK5-AS1 could sponge miR-154-5p in HCC cells.

Previous studies have reported that pleomorphic adenoma gene like-2 (PLAGL2), as a member of the PLAG zinc finger transcription factor, is widely involved in the progress of various tumors [40-42], including HCC. For example, Liang $\mathrm{Wu}$ et al. confirmed that PLAGL2 promoted epithelial-mesenchymal transition and mediated colorectal cancer metastasis via $\beta$-catenin-dependent regulation of ZEB1 [42]. And Weiwei $\mathrm{Hu}$ et al. found that PLAGL2 regulated the transcription of EGFR, enhanced the PI3K/PDK1/mTORC2-dependent AKT phosphorylation, and upregulated expression of HIF-1/ $2 \alpha[34,43]$. In the present study, we discovered a new mechanism regulating PLAGL2 expression at the posttranscriptional level under hypoxia. Firstly, we identified PLAGL2 as the direct target of miR-154-5p in HCC cells through bioinformatic prediction and experimental verification. Secondly, we found that PLAGL2 was positively modulated by MAPKAPK5-AS1 in HCC cells. Thirdly, we found the expression of PLAGL2 was negatively correlated with miR-154-5p expression while positively correlated with MAPKAPK5-AS1 expression in HCC tissues. Finally, we conducted a series of recusing assays to illustrate that PLAGL2 mediated the oncogenic function of MAPKAPK5-AS1 in HCC cells. Taken together, we demonstrated that MAPKAPK5-AS1 could promote PLAGL2 expression by sponging miR-154-5p in HCC.

Hypoxia, a significant feature of solid tumors, widely affects various tumors [44-46]. Studies have found that many hypoxia-responsive lncRNAs are involved in the impact of hypoxia on tumor biological behavior. For example, Aijun Yu et al. confirmed that the lncRNA H19/ microRNA-612/Bcl-2 axis mediates the promotion of HIF- $1 \alpha$ on proliferation, migration, and invasion in cholangiocarcinoma [47]. In the current study, we investigated the effect of hypoxia on MAPKAPK5-AS1 expression for the first time. Firstly, we observed that hypoxia could induce MAPKAPK5-AS1 expression, which could be significantly impaired by HIF-1 $\alpha$ knockdown. Subsequently, we predicted a potential HRE in the promoter of MAPKAPK5-AS1 through the JASPAR 
database. Moreover, we confirmed the regulatory effect of HIF- $1 \alpha$ on MAPKAPK5-AS1 transcription in response to hypoxia by ChIP and dual-luciferase reporter assays. Next, our results revealed that the upregulation of HIF- $1 \alpha$ and PLAGL2 induced by hypoxia was obviously attenuated by MAPKAPK5-AS1 knockdown, and hypoxia elevated the level of HIF- $1 \alpha$ and MAPKAPK5AS1, which could be partly reversed by PLAGL2 knockdown. These results indicated that HIF-1 $\alpha$, as a transcript factor, could directly bind to the MAPK APK5-AS1 promoter to activate its transcription, and MAPKAPK5-AS1 regulates HIF-1 $\alpha$ expression through PLAGL2 to form a hypoxia-mediated MAPKAPK5-AS1/ PLAGL2/HIF-1 $\alpha$ signaling loop in HCC (Fig. 10).

\section{Conclusions}

In the current study, we identified an upregulated lncRNA MAPKAPK5-AS1, associated with poor clinical features and prognosis of HCC. Moreover, MAPKAPK5AS1 contributed to the growth and metastasis of HCC cells through the miR-154-5p/PLAGL2/EGRT/AKT axis. Furthermore, our results indicate that HIF- $1 \alpha$, as a transcript factor, could directly bind to the MAPKAPK5AS1 promoter to activate its transcription, and MAPK APK5-AS1 regulates HIF-1 $\alpha$ expression through PLAG L2 to form a hypoxia-mediated MAPKAPK5-AS1/ PLAGL2/HIF- $1 \alpha$ signaling loop in HCC. In summary, the study reveals the role of MAPKAPK5-AS1 in HCC progression, and it could be a potential therapeutic target and prognostic predictor of HCC.

\section{Supplementary Information}

The online version contains supplementary material available at https:/doi. org/10.1186/s13046-021-01868-z.

\section{Additional file 1. \\ Additional file 2. \\ Additional file 3: Figure S1. The expression and clinical significance of MAPKAPK5-AS1 in HCC. (A) GSE54236 dataset showed the high expression level of MAPKAPK5-AS1 in HCC tissues compared to normal tissues. (B) The level of MAPKAPK5-AS1 in human normal liver cell (L02) and HCC cell lines. (C) The association between MAPKAPK5-AS1 expression and pathological stage of HCC patients. (D) The association between MAPKAPK5-AS1 expression and tumor grade of HCC patients. ${ }^{*} p<0.05$.}

Additional file 4: Figure S2. Transfection efficiency of these four HCC cell lines with MAPKAPK5-AS1 overexpression or knockdown. ${ }^{*} p<0.05$.

Additional file 5: Figure S3. The level of MAPKAPK5-AS1 in subcutaneous tumor tissues. ${ }^{*} p<0.05$.

Additional file 6: Figure S4. Prediction of targeted miRNAs for MAPK APK5-AS1. (A) Subcellular fractionation assay was applied to determine MAPKAPK5-AS1 subcellular localization in Hep3B and Huh-7 cells. (B) The level of miR-1271-5p, miR-154-5p, and miR-450b-5p in HCCLM3 and MHCC97-H cells after MAPKAPK5-AS1 knockdown. (C) TCGA data accessed via ENCORI online platform showed the low miR-154-5p expression in HCC. ${ }^{*} p<0.05$

Additional file 7: Figure S5. MiR-154-5p mediates the function of MAPKAPK5-AS1. (A-C) MTT, colony formation and EdU assays were conducted to explore the effect of miR-154-5p mimics on cell proliferation enhanced by MAPKAPK5-AS1. (D) Flow cytometry showed the effect of miR-154-5p mimics on apoptosis inhibited by MAPKAPK5-AS1. (E-F) Transwell assay were performed to evaluate the effects of miR-154-5p mimics on the tumor cells abilities of migration and invasion enhanced by MAPKAPK5-AS1. (C-D) Expression of EMT markers was detected by western blotting to assess the effect of miR-154-5p mimics on EMT process promoted by MAPKAPK5-AS1. ${ }^{*} p<0.05$.

Additional file 8: Figure S6. The level of PLAGL2 in HCC tissues and subcutaneous tumor tissues. (A-B) The effect of miR-154-5p on PLAGL2 mRNA level evaluated by qRT-PCR. (C) TCGA data accessed via ENCORI online platform showed the high PLAGL2 expression in HCC. (D) TCGA data accessed via ENCORI online platform showed the positive association between PLAGL2 and MAPKAPK5-AS1 in HCC. (E) The PLAGL2 expression in xenograft tumors.

Additional file 9: Figure S7. PLAGL2 mediates the oncogenic effect of MAPKAPK5-AS1. (A-C) MTT, colony formation and EdU assays showed the effect of PLAGL2 kncokdown on cell proliferation enhanced by MAPK APK5-AS1. (D) Flow cytometry showed the effect of PLAGL2 kncokdown on cell apoptosis inhibited by MAPKAPK5-AS1. (E) Transwell assay showed the effects of PLAGL2 kncokdown on migration and invasion of the tumor cells promoted by MAPKAPK5-AS1. (F) Expression of EMT and EGFR/AKT pathway markers was detected by western blotting to assess the effect of PLAGL2 knockdown on EMT process and pathway activity promoted by MAPKAPK5-AS1. ${ }^{*} p<0.05$.

Additional file 10: Figure S8. EGFR/AKT pathway mediates the oncogenic effect of MAPKAPK5-AS1. (A-B) MTT and EdU assays showed the the effect of MK2206, the inhibitor of AKT, on cell proliferation enhanced by MAPKAPK5-AS1. (C) Flow cytometry showed the effect of MK2206 on cell apoptosis inhibited by MAPKAPK5-AS1. (D) Transwell assay showed the effects of MK2206 on migration and invasion of the tumor cells promoted by MAPKAPK5-AS1. (E) Expression of EMT markers was detected by western blotting to assess the effect of MK2206 on EMT process promoted by MAPKAPK5-AS1. ${ }^{*} p<0.05$.

\section{Abbreviations}

HCC: Hepatocellular carcinoma; PLAGL2: Pleomorphic adenoma gene like-2; EGFR: Epidermal growth factor receptor; HIF: Hypoxia-inducible factor; EMT: Epithelial-mesenchymal transition; miRNA: MicroRNAs; 3'UTR: 3'Untranslated regions; ceRNA: Competitive endogenous RNA; TCGA: The cancer genome atlas; GEO: Gene expression omnibus; FBXW7: F-box and WD repeat domain containing 7; FOXA1: Forkhead box A1; RIP: RNA immunoprecipitation; ChIP: Chromatin immunoprecipitation

\section{Acknowledgements}

Not applicable.

\section{Authors' contributions}

QL and KT conceived and designed the experiments; LW, LS, RL, HM, TC, and YN performed the experiments; LW, RL and TC analyzed the data; YW and SH contributed reagents/materials/analysis tools; WL, QL and KT wrote the paper. All authors read and approved the final manuscript.

\section{Funding}

This study was supported by grants from the National Natural Science Foundation of China $(81874069,82072680)$, Nature Science Basic Research. Program of Shaanxi (No. 2019JM-133, 2020JC-36), and Fundamental Research Funds for the Central Universities (No. xtr042019011).

\section{Availability of data and materials}

All data generated or analyzed during this study are included either in this article or in the supplementary information files.

\section{Ethics approval and consent to participate}

All procedures performed in studies involving human participants were in accordance with the ethical standards of the Research Ethics Committee of The First Affiliated Hospital of Xi'an Jiaotong University and with the 1964 Helsinki declaration and its later amendments. All written informed consent 
to participate in the study was obtained from HCC patients for samples to be collected from them.

\section{Consent for publication}

Not applicable.

\section{Competing interests}

The authors declare that they have no competing interests.

Received: 5 December 2020 Accepted: 5 February 2021

Published online: 17 February 2021

\section{References}

1. Llovet JM, Villanueva A, Lachenmayer A, Finn RS. Advances in targeted therapies for hepatocellular carcinoma in the genomic era. Nat Rev Clin Oncol. 2015;12:408-24.

2. Goh GB, Chang PE, Tan CK. Changing epidemiology of hepatocellular carcinoma in Asia. Best Pract Res Clin Gastroenterol. 2015;29:919-28.

3. Mu M, Niu W, Zhang $X$, Hu S, Niu C. LncRNA BCYRN1 inhibits glioma tumorigenesis by competitively binding with miR-619-5p to regulate CUEDC2 expression and the PTEN/AKT/p21 pathway. Oncogene. 2020

4. Z Zhang X, Niu W, Mu M, Hu S, Niu C. Long non-coding RNA LPP-AS2 promotes glioma tumorigenesis via miR-7-5p/EGFR/PI3K/AKT/C-MYC feedback loop. J Exp Clin Cancer Res. 2020;39:196.

5. Zhang J, Qiu WQ, Zhu H, Liu H, Sun JH, Chen Y, Shen H, Qian CL, Shen ZY. HOTAIR contributes to the carcinogenesis of gastric cancer via modulating cellular and exosomal miRNAs level. Cell Death Dis. 2020;11:780.

6. Li Z, Lu X, Liu Y, Zhao J, Ma S, Yin H, Huang S, Zhao Y, He X. Gain of LINC00624 enhances liver cancer progression by disrupting the HDAC6TRIM28-ZNF354C Corepressor complex. Hepatology. 2020. https:/doi.org/ 0.1002/hep.31530. Online ahead of print.

7. He H, Wang Y, Ye P, Yi D, Cheng Y, Tang H, Zhu Z, Wang X, Jin S. Long noncoding RNA ZFPM2-AS1 acts AS a miRNA sponge and promotes cell invasion through regulation of miR-139/GDF10 in hepatocellular carcinoma. J Exp Clin Cancer Res. 2020;39:159.

8. Wang Y, Liu Z, Yao B, Li Q, Wang L, Wang C, Dou C, Xu M, Liu Q, Tu K. Long non-coding RNA CASC2 suppresses epithelial-mesenchymal transition of hepatocellular carcinoma cells through CASC2/miR-367/FBXW7 axis. Mol Cancer. 2017:16:123.

9. Wang Y, Yang L, Chen T, Liu X, Guo Y, Zhu Q, Tong X, Yang W, Xu Q, Huang D, Tu K. A novel InCRNA MCM3AP-AS1 promotes the growth of hepatocellular carcinoma by targeting miR-194-5p/FOXA1 axis. Mol Cancer. 2019;18:28.

10. Wang Y, Sun L, Wang L, Liu Z, Li Q, Yao B, Wang C, Chen T, Tu K, Liu Q. Long non-coding RNA DSCR8 acts as a molecular sponge for miR-485-5p to activate Wnt/beta-catenin signal pathway in hepatocellular carcinoma. Cell Death Dis. 2018:9:851.

11. Liu Z, Wang Y, Wang L, Yao B, Sun L, Liu R, Chen T, Niu Y, Tu K, Liu Q. Long non-coding RNA AGAP2-AS1, functioning AS a competitive endogenous RNA, upregulates ANXA11 expression by sponging miR-16-5p and promotes proliferation and metastasis in hepatocellular carcinoma. J Exp Clin Cancer Res. 2019:38:194.

12. Sun L, Wang L, Chen T, Shi Y, Yao B, Liu Z, Wang Y, Li Q, Liu R, Niu Y, et al. LnCRNA RUNX1-IT1 which is downregulated by hypoxia-driven histone deacetylase 3 represses proliferation and cancer stem-like properties in hepatocellular carcinoma cells. Cell Death Dis. 2020;11:95.

13. Liu Z, Mo H, Sun L, Wang L, Chen T, Yao B, Liu R, Niu Y, Tu K, Xu Q, Yang N. Long noncoding RNA PICSAR/miR-588/EIF6 axis regulates tumorigenesis of hepatocellular carcinoma by activating PI3K/AKT/mTOR signaling pathway. Cancer Sci. 2020;111:4118-28.

14. Liu Y, Wang X, Li W, Xu Y, Zhuo Y, Li M, He Y, Wang X, Guo Q, Zhao L, Qiang L. Oroxylin a reverses hypoxia-induced cisplatin resistance through inhibiting HIF-1alpha mediated XPC transcription. Oncogene. 2020;39:6893-905

15. Shimamoto K, Tanimoto K, Fukazawa T, Nakamura H, Kanai A, Bono H, Ono H, Eguchi H, Hirohashi N. GLIS1, a novel hypoxia-inducible transcription factor, promotes breast cancer cell motility via activation of WNT5A. Carcinogenesis. 2020;41:1184-94.

16. Yuen WW, Wong CC. Hypoxia-inducible factors and innate immunity in liver cancer. J Clin Invest. 2020;130:5052-62.
17. Wang N, Shi XF, Khan SA, Wang B, Semenza GL, Prabhakar NR, Nanduri J. Hypoxia inducible factor-1 mediates pancreatic beta-cell dysfunction by intermittent hypoxia. Am J Physiol Cell Physiol. 2020;319:C922-32.

18. Wang Z, Cui M, Qu Y, He R, Wu W, Lin H, Shao Z. Hypoxia protects rat bone marrow Mesenchymal stem cells against compression-induced apoptosis in the degenerative disc microenvironment through activation of the HIF1alpha/YAP signaling pathway. Stem Cells Dev. 2020

19. Sun CC, Zhu W, Li SJ, Hu W, Zhang J, Zhuo Y, Zhang H, Wang J, Zhang Y, Huang SX, et al. FOXC1-mediated LINC00301 facilitates tumor progression and triggers an immune-suppressing microenvironment in non-small cell lung cancer by regulating the HIF1alpha pathway. Genome Med. 2020;12:77.

20. Zhang JH, Shen Y, Liu C, Yang J, Yang YQ, Zhang C, Bian SZ, Yu J, Gao XB, Zhang LP, et al. EPAS1 and VEGFA gene variants are related to the symptoms of acute mountain sickness in Chinese Han population: a crosssectional study. Mil Med Res. 2020;7:35.

21. Qiu L, Zhao Q, Dai L, Zhu A, Xu X, Zhao S, Chen J. Long non-coding RNA DANCR alleviates hypoxia-caused $\mathrm{H} 9 \mathrm{c} 2$ cells damage through up regulation of HIF-1alpha. Artif Cells Nanomed Biotechnol. 2020;48:533-41.

22. Dong $L$, Cao X, Luo Y, Zhang G, Zhang D. A positive feedback loop of IncRNA DSCR8/miR-98-5p/STAT3/HIF-1alpha plays a role in the progression of ovarian Cancer. Front Oncol. 2020:10:1713.

23. Hu M, Fu Q, Jing C, Zhang X, Qin T, Pan Y. LncRNA HOTAIR knockdown inhibits glycolysis by regulating miR-130a-3p/HIF1A in hepatocellular carcinoma under hypoxia. Biomed Pharmacother. 2020;125:109703.

24. Wang $L$, Mo $H$, Jiang $Y$, Wang $Y$, Sun L, Yao B, Chen T, Liu R, Li Q, Liu Q, Yin G. MicroRNA-519c-3p promotes tumor growth and metastasis of hepatocellular carcinoma by targeting BTG3. Biomed Pharmacother. 2019; 118:109267

25. Lin MF, Yang YF, Peng ZP, Zhang MF, Liang JY, Chen W, Liu XH, Zheng YL. FOXK2, regulted by miR-1271-5p, promotes cell growth and indicates unfavorable prognosis in hepatocellular carcinoma. Int J Biochem Cell Biol. 2017;88:155-61.

26. Liu HM, Tan HY, Lin Y, Xu BN, Zhao WH, Xie YA. MicroRNA-1271-5p inhibits cell proliferation and enhances radiosensitivity by targeting CDK1 in hepatocellular carcinoma. J Biochem. 2020;167:513-24.

27. Chen J, Ma C, Zhang Y, Pei S, Du M, Zhang Y, Oian L, Wang J, Yin L, He X MiR-154-5p suppresses cell invasion and migration through inhibiting KIF14 in nasopharyngeal carcinoma. Onco Targets Ther. 2020;13:2235-46.

28. Wang $X$, Sun S, Tong $X$, Ma Q, Di H, Fu T, Sun Z, Cai Y, Fan W, Wu Q, et al MiRNA-154-5p inhibits cell proliferation and metastasis by targeting PIWIL1 in glioblastoma. Brain Res. 1676;2017:69-76.

29. Zheng Y, Zhu C, Ma L, Shao P, Qin C, Li P, Cao Q, Ju X, Cheng G. Zhu Q, et al: miRNA-154-5p inhibits proliferation, migration and invasion by targeting E2F5 in prostate Cancer cell lines. Urol Int. 2017;98:102-10.

30. Han H, Shao Q, Liu X. LINC00441 promotes cervical cancer progression by modulating miR-450b-5p/RAB10 axis. Cancer Cell Int. 2020;20:368.

31. Li H, Shen S, Chen X, Ren Z, Li Z, Yu Z. miR-450b-5p loss mediated KIF26B activation promoted hepatocellular carcinoma progression by activating PI3KJAKT pathway. Cancer Cell Int. 2019:19:205.

32. Zhang Q, Cao LY, Cheng SJ, Zhang AM, Jin XS, Li Y. p53-induced microRNA1246 inhibits the cell growth of human hepatocellular carcinoma cells by targeting NFIB. Oncol Rep. 2015;33:1335-41

33. Jiang D, Hu B, Wei L, Xiong Y, Wang G, Ni T, Zong C, Ni R, Lu C. High expression of vacuolar protein sorting $4 B$ (VPS4B) is associated with accelerated cell proliferation and poor prognosis in human hepatocellular carcinoma. Pathol Res Pract. 2015;211:240-7.

34. Hu W, Zheng $\mathrm{S}$, Guo H, Dai B, Ni J, Shi Y, Bian H, Li L, Shen Y, Wu M, et al. PLAGL2-EGFR-HIF-1/2alpha signaling loop promotes HCC progression and Erlotinib insensitivity. Hepatology. 2020. https://doi.org/10.1002/hep.31293. Online ahead of print.

35. Wang C, Zi H, Wang Y, Li B, Ge Z, Ren X. LncRNA CASC15 promotes tumour progression through SOX4/Wnt/beta-catenin signalling pathway in hepatocellular carcinoma. Artif Cells Nanomed Biotechnol. 2020;48:763-9.

36. Sun Z, Xue S, Zhang M, Xu H, Hu X, Chen S, Liu Y, Guo M, Cui H. Aberrant NSUN2-mediated $m$ (5) C modification of H19 InCRNA is associated with poor differentiation of hepatocellular carcinoma. Oncogene. 2020;39:6906-19.

37. Chen BW, Zhou Y, Wei T, Wen L, Zhang YB, Shen SC, Zhang J, Ma T, Chen W, Ni L, et al. IncRNA-POIR promotes epithelial-mesenchymal transition and suppresses sorafenib sensitivity simultaneously in hepatocellular carcinoma by sponging miR-182-5p. J Cell Biochem. 2021;122:130-42. 
38. Ji H, Hui B, Wang J, Zhu Y, Tang L, Peng P, Wang T, Wang L, Xu S, Li J, Wang K. Long noncoding RNA MAPKAPK5-AS1 promotes colorectal cancer proliferation by partly silencing p21 expression. Cancer Sci. 2019;110:72-85.

39. Yang Z, Jiang S, Shang J, Jiang Y, Dai Y, Xu B, Yu Y, Liang Z, Yang Y. LncRNA: shedding light on mechanisms and opportunities in fibrosis and aging. Ageing Res Rev. 2019;52:17-31.

40. Zhao Z, Shelton SD, Oviedo A, Baker AL, Bryant CP, Omidvarnia S, Du L. The PLAGL2/MYCN/miR-506-3p interplay regulates neuroblastoma cell fate and associates with neuroblastoma progression. J Exp Clin Cancer Res. 2020;39:41.

41. Zhou Z, Wu L, Liu Z, Zhang X, Han S, Zhao N, Bao H, Yuan W, Chen J, Ji J, Shu X. MicroRNA-214-3p targets the PLAGL2-MYH9 axis to suppress tumor proliferation and metastasis in human colorectal cancer. Aging (Albany NY). 2020;12:9633-57.

42. Cao Y, Tao Q, Kao X, Zhu X. Hsa-circRNA-103809 promotes hepatocellular carcinoma development via MicroRNA-1270/PLAG1 like zinc finger 2 Axis. Dig Dis Sci. 2020.

43. Wu L, Zhou Z, Han S, Chen J, Liu Z, Zhang X, Yuan W, Ji J, Shu X. PLAGL2 promotes epithelial-mesenchymal transition and mediates colorectal cancer metastasis via beta-catenin-dependent regulation of ZEB1. Br J Cancer. 2020;122:578-89.

44. Niklasson CU, Fredlund E, Monni E, Lindvall JM, Kokaia Z, Hammarlund EU, Bronner ME, Mohlin S. Hypoxia inducible factor-2alpha importance for migration, proliferation, and self-renewal of trunk neural crest cells. Dev Dyn. 2021;250:191-236.

45. Hsin-Fu Liu O, Kiema M, Beter M, Yla-Herttuala S, Laakkonen JP, Kaikkonen MU. Hypoxia-mediated regulation of histone demethylases affects angiogenesis-associated functions in endothelial cells. Arterioscler Thromb Vasc Biol. 2020;40:2665-77.

46. Renfrow JJ, Soike MH, West JL, Ramkissoon SH, Metheny-Barlow L, Mott RT, Kittel CA, D'Agostino RB Jr, Tatter SB, Laxton AW, et al. Attenuating hypoxia driven malignant behavior in glioblastoma with a novel hypoxia-inducible factor 2 alpha inhibitor. Sci Rep. 2020;10:15195.

47. Yu A, Zhao L, Kang Q, Li J, Chen K, Fu H. Transcription factor HIF1alpha promotes proliferation, migration, and invasion of cholangiocarcinoma via long noncoding RNA H19/microRNA-612/BCl-2 axis. Transl Res. 2020; 224:26-39.

\section{Publisher's Note}

Springer Nature remains neutral with regard to jurisdictional claims in published maps and institutional affiliations.

Ready to submit your research? Choose BMC and benefit from:

- fast, convenient online submission

- thorough peer review by experienced researchers in your field

- rapid publication on acceptance

- support for research data, including large and complex data types

- gold Open Access which fosters wider collaboration and increased citations

- maximum visibility for your research: over $100 \mathrm{M}$ website views per year

At $\mathrm{BMC}$, research is always in progress.

Learn more biomedcentral.com/submissions 